\title{
A planetary system with two transiting mini-Neptunes near the radius valley transition around the bright $\mathrm{M}$ dwarf TOI-776 ${ }^{\star}$
}

\author{
R. Luque ${ }^{1,2}$, L. M. Serrano ${ }^{3}$, K. Molaverdikhani ${ }^{4,5}$, M. C. Nixon ${ }^{6}$, J. H. Livingston ${ }^{7}$, E. W. Guenther ${ }^{8}$, E. Pallé , $^{1,2}$, \\ N. Madhusudhan ${ }^{6}$, G. Nowak ${ }^{1,2}$, J. Korth ${ }^{9}$, W. D. Cochran ${ }^{10}$, T. Hirano ${ }^{11}$, P. Chaturvedi ${ }^{8}$, E. Goffo ${ }^{3}$, S. Albrecht ${ }^{12}$, \\ O. Barragán ${ }^{13}$, C. Briceño ${ }^{14}$, J. Cabrera ${ }^{15}$, D. Charbonneau ${ }^{16}$, R. Cloutier ${ }^{16}$, K. A. Collins ${ }^{16}$, K. I. Collins ${ }^{17}$, \\ K. D. Colón ${ }^{18}$, I. J. M. Crossfield ${ }^{19}$, Sz. Csizmadia ${ }^{15}$, F. Daii ${ }^{20}$, H. J. Deeg ${ }^{1,2}$, M. Esposito ${ }^{8}$, M. Fridlund ${ }^{21,22}$, \\ D. Gandolfi ${ }^{3}$, I. Georgieva ${ }^{22}$, A. Glidden ${ }^{23,24}$, R. F. Goeke ${ }^{23}$, S. Grziwa ${ }^{9}$, A. P. Hatzes ${ }^{8}$, C. E. Henze ${ }^{25}$, \\ S. B. Howell ${ }^{25}$, J. Irwin ${ }^{16}$, J. M. Jenkins ${ }^{25}$, E. L. N. Jensen ${ }^{26}$, P. Kábath ${ }^{27}$, R. C. Kidwell Jr. ${ }^{28}$, J. F. Kielkopf ${ }^{29}$, \\ E. Knudstrup ${ }^{12}$, K. W. F. Lam ${ }^{30}$, D. W. Latham ${ }^{16}$, J. J. Lissauer ${ }^{25}$, A. W. Mann ${ }^{31}$, E. C. Matthews ${ }^{24}$, I. Mireles ${ }^{24}$, \\ N. Narita ${ }^{32,33,34,1}$, M. Paegert ${ }^{16}$, C. M. Persson ${ }^{22}$, S. Redfield ${ }^{35}$, G. R. Ricker ${ }^{24}$, F. Rodler ${ }^{36}$, J. E. Schlieder ${ }^{18}$, \\ N. J. Scott ${ }^{25}$, S. Seager ${ }^{24,23,37}$, J. Šubjak ${ }^{27}$, T. G. Tan ${ }^{38}$, E. B. Ting ${ }^{25}$, R. Vanderspek ${ }^{24}$, V. Van Eylen ${ }^{39}$, \\ J. N. Winn ${ }^{40}$, and C. Ziegler ${ }^{41}$
}

(Affiliations can be found after the references)

Received 16 September 2020 / Accepted 30 November 2020

\begin{abstract}
We report the discovery and characterization of two transiting planets around the bright M1 V star LP 961-53 (TOI-776, $J=8.5 \mathrm{mag}$, $M=0.54 \pm 0.03 M_{\odot}$ ) detected during Sector 10 observations of the Transiting Exoplanet Survey Satellite (TESS). Combining the TESS photometry with HARPS radial velocities, as well as ground-based follow-up transit observations from the MEarth and LCOGT telescopes, for the inner planet, TOI-776 b, we measured a period of $P_{\mathrm{b}}=8.25 \mathrm{~d}$, a radius of $R_{\mathrm{b}}=1.85 \pm 0.13 R_{\oplus}$, and a mass of $M_{\mathrm{b}}=4.0 \pm 0.9 M_{\oplus}$; and for the outer planet, TOI-776 c, a period of $P_{\mathrm{c}}=15.66 \mathrm{~d}$, a radius of $R_{\mathrm{c}}=2.02 \pm 0.14 R_{\oplus}$, and a mass of $M_{\mathrm{c}}=5.3 \pm 1.8 M_{\oplus}$. The Doppler data shows one additional signal, with a period of $\sim 34 \mathrm{~d}$, associated with the rotational period of the star. The analysis of fifteen years of ground-based photometric monitoring data and the inspection of different spectral line indicators confirm this assumption. The bulk densities of TOI-776 b and c allow for a wide range of possible interior and atmospheric compositions. However, both planets have retained a significant atmosphere, with slightly different envelope mass fractions. Thanks to their location near the radius gap for $\mathrm{M}$ dwarfs, we can start to explore the mechanism(s) responsible for the radius valley emergence around low-mass stars as compared to solar-like stars. While a larger sample of well-characterized planets in this parameter space is still needed to draw firm conclusions, we tentatively estimate that the stellar mass below which thermally-driven mass loss is no longer the main formation pathway for sculpting the radius valley is between 0.63 and $0.54 M_{\odot}$. Due to the brightness of the star, the TOI-776 system is also an excellent target for the James Webb Space Telescope, providing a remarkable laboratory in which to break the degeneracy in planetary interior models and to test formation and evolution theories of small planets around low-mass stars.
\end{abstract}

Key words. planetary systems - techniques: photometric - techniques: radial velocities - stars: individual: LP 961-53 stars: low-mass

\section{Introduction}

Exoplanets with masses between those of Earth and Uranus are characterized by a broad range of measured bulk densities (e.g., Hatzes \& Rauer 2015). A low density suggests the presence of an extended $\mathrm{H} / \mathrm{He}$-envelope around a solid core. On the contrary, if the density is high, the exoplanet is considered to be fully rocky or enriched in light elements (e.g., water, methane, ammonia). The absence of an envelope might be the result of two opposite scenarios: the planet is born without it, or the planet loses it over time. In the first case, the planet forms in a gas-poor inner protoplanetary disk without a thick H/He-envelope (e.g., Lee et al. 2014; Lee \& Chiang 2016). For the second case, different mechanisms have been proposed in the last years, such as slow atmospheric escape powered by the planetary core's primordial energy reservoir from formation (Ginzburg et al. 2018;

\footnotetext{
* Based on observations made with ESO Telescopes at the La Silla Observatory under programs ID 1102.C-0923 and 60.A-9709.
}

Gupta \& Schlichting 2019, 2020), impact erosion by planetesimals (Shuvalov 2009; Schlichting et al. 2015; Wyatt et al. 2020), or erosion processes driven by the stellar X-ray+EUV (XUV) radiation (e.g., Murray-Clay et al. 2009; Lammer et al. 2012; Owen \& Jackson 2012; Owen \& Wu 2013; Kislyakova et al. 2013, 2014; Lopez \& Fortney 2014; Jin et al. 2014; Chen \& Rogers 2016; Osborn et al. 2017; Jin \& Mordasini 2018; Lopez \& Rice 2018; Wu 2019; Mordasini 2020).

For the latter, the erosion rate increases if the planetary surface gravity decreases and the amount of XUV radiation that the planet receives increases. In addition, the intensity of XUVradiation depends on the orbital semi-major axis and on the stellar activity level. The XUV-radiation is particularly high at young ages, and then it declines as a result of age, mass, and stellar rotation (Walter et al. 1988; Briceno et al. 1997; Tu et al. 2015). A star that begins its life rapidly rotating will suffer a more rapid decline in rotation than a star that was initially a slow rotator. Thus, for a star of several Gyr, understanding its 
original activity level is challenging. The presence, or absence, of a hydrogen-rich envelope in a system containing just one planet can thus equally be explained by assuming that the host star was either a slow or rapid rotator when it was young. Systems containing more than one planet are necessary to test the theory of atmospheric erosion, because the origin of all the planets of a system should be explained with a unique evolutionary history of the host's XUV radiation (Owen \& Campos Estrada 2020).

On the other hand, the amount of XUV radiation also depends on the stellar type. The XUV luminosities of young G and $\mathrm{M}$ stars are similar to each other. The average X-ray luminosity of $\mathrm{G}$ stars is $10^{29} \mathrm{erg} \mathrm{s}^{-1}$, while in the case of $\mathrm{M}$ dwarfs, the $50 \mathrm{Myr}$ stars in $\alpha$-Per, for example, have luminosities of $10^{28} \mathrm{erg} \mathrm{s}^{-1}$ (France et al. 2016). The main difference is that M dwarfs remain in the high activity phase for up to $2 \mathrm{Gyr}$ (Johnstone et al. 2015), a much longer amount of time compared to the $300 \mathrm{Myr}$ of G-stars (Güdel et al. 2004). This makes $\mathrm{M}$ dwarfs preferred targets to study planetary systems that have experienced significant stellar XUV irradiation. Another advantage of $\mathrm{M}$ dwarfs is their small size, which makes it easier to detect smaller transiting planets. The paucity of close-in planets around mid-K to mid-M dwarfs between approximately 1.4 and $1.7 R_{\oplus}$ (Cloutier et al. 2020), known as the radius valley, marks the transition between rocky planets and sub-Neptunes orbiting low-mass stars. As such, M dwarfs' multi-planetary systems, which include sub-Neptunes and/or rocky planets, represent an ideal benchmark for testing the theory of atmospheric erosion.

Gas-poor formation provides an alternative to explain the absence of $\mathrm{H} / \mathrm{He}$ envelopes in some low-mass planets, since the erosion scenario presents some issues. For instance, if a close-in $10 M_{\oplus}$ rocky planet forms while there is still a gaseous disk, its mass is high enough to undergo runaway accretion and become a Jupiter-type planet. The detection of close-in Jupiter-mass planets, at least in A stars, shows that it is hard to reconstruct a mechanism that transforms a Jupiter into a rocky super-Earth, since any working physical process should be able to completely strip off the $\mathrm{H} / \mathrm{He}$ atmosphere. On the other hand, stars hosting hot Jupiters have high metallicities, while rocky planets are equally distributed between metal-poor and metal-rich stars (Winn et al. 2017). Thus, there are two alternative scenarios within gas-poor formation models that could explain the existence of rocky super-Earths. Either the dust-to-gas ratio of the inner disk is 20-times higher than solar, or the gas accretion is delayed until just before the disk disperses (Lee et al. 2014; Lee \& Chiang 2016).

Lopez \& Rice (2018) proposed a statistical test that could allow us to understand the most likely formation history for super-Earths. If a high percentage of rocky planets are the evaporated cores of sub-Neptunes, the transition radius from rocky to sub-Neptune planets should decrease for longer orbital periods. On the contrary, if the gas-poor formation scenario is correct, the transition radius should increase with orbital period. Another methodology to test the formation theory of superEarths requires studying the position of the radius valley for stars with different masses, thus of different stellar types. If the photoevaporation scenario is correct, the radius valley shifts toward planets of smaller radii for stars of lower masses. If, on the contrary, the gas-poor formation scenario is at work, the valley position is not affected by the stellar mass (Cloutier \& Menou 2020). However, since the radius valley represents the range of radii in which the transition between rocky planets and sub-Neptunes occurs, it is necessary to accurately determine the mass and radius of the planets to calculate the mass-fraction of their envelope and unveil their nature. Therefore, the ideal test to understand which model is more realistic between the gaspoor formation and the photoevaporation consists of measuring the masses and radii of the planets close to, or inside, the radius valley, preferably in a multi-planetary system around low-mass stars. Therefore, we can also constrain these models in a much better way than through the radius distribution alone.

As of today, there is a limited number of known multiplanetary systems that orbit M dwarfs $\left(3000 \mathrm{~K}<T_{\text {eff }}<4000 \mathrm{~K}\right.$; as a proxy of M0 V-M5 V, Cifuentes et al. 2020) and respect the condition $\left(M_{\mathrm{p}}<10 M_{\oplus}\right)$ required to test the two mentioned formation theories. There are only two systems with three transiting planets with measured dynamical masses, Kepler-138 (Almenara et al. 2018) and L 98-59 (Cloutier et al. 2019), and four systems with two transiting planets: LHS 1140 (Lillo-Box et al. 2020), LTT 3780 (Nowak et al. 2020; Cloutier et al. 2020), K2-146 (Lam et al. 2020; Hamann et al. 2019), and Kepler-26 (JontofHutter et al. 2016). This paucity of systems is inadequate for understanding the formation and evolution of planetary systems around $\mathrm{M}$ dwarfs. The discovery of each new system is thus important, especially if the host star is bright and the planets are close to the radius valley.

In this paper, we present the discovery of two transiting planets orbiting an M1 V star. The inner one has a period of $8.2 \mathrm{~d}$ and a radius of $\sim 1.8 R_{\oplus}$; thus, it is close to the radius valley. The outer planet has a period of $15.7 \mathrm{~d}$ and a radius of $2.0 R_{\oplus}$ in the subNeptune regime. By measuring their masses, we explore whether these new planets are characterized by extended $\mathrm{H} / \mathrm{He}$ envelopes. Since they orbit a relatively bright, nearby $M$ dwarf, these new objects represent ideal targets for follow-up atmospheric studies.

\section{TESS photometry}

The star LP 961-53 (TIC 306996324) was observed with TESS in Sector 10 (Camera \#2, CCD \#4) from March 26, 2019 until April 22, 2019, with 2-min cadence exposures, and it will be observed again in Sector 37 from April 2 to 28, 2021. Data collection was paused for $0.98 \mathrm{~d}$ during perigee passage, while downloading data. The Science Processing Operations Center (SPOC; Jenkins et al. 2016) at the NASA Ames Research Center made the data available at the Mikulski Archive for Space Telescopes $(\mathrm{MAST})^{1}$ on June 1, 2019. SPOC provided simple aperture photometry (SAP) for this target as well as systematicscorrected photometry, a procedure consisting of an adaptation of the Kepler Presearch Data Conditioning algorithm (PDC; Smith et al. 2012; Stumpe et al. 2012, 2014) to TESS. Figure 1 shows the TESS pixels included in the computation of the SAP and PDCcorrected SAP data. For the remainder of this work, we make use of the latter photometric data, shown in Fig. 2.

On June 11, 2019, two transiting candidates orbiting LP 96153 were announced in the TESS data public website ${ }^{2}$ under the TESS Object of Interest (TOI) number 776. TOI-776.01 is a planet candidate with a period of $15.65 \mathrm{~d}$, a transit depth of $1484 \pm 127 \mathrm{ppm}$, and an estimated planet radius of $2.2 \pm 0.6 R_{\oplus}$; while TOI-776.02 is a planet candidate with a period of $8.24 \mathrm{~d}$, a transit depth of $1063 \pm 104 \mathrm{ppm}$, and an estimated planet radius of $1.8 \pm 1.4 R_{\oplus}$. Both candidates passed all the tests from the threshold crossing event (TCE) Data Validation Report (DVR; Twicken et al. 2018; Li et al. 2019): even-odd transits comparison, eclipsing binary (EB) discrimination tests, ghost diagnostic tests to help rule out scattered light, or background EB, among

\footnotetext{
https://mast.stsci.edu

https://tev.mit.edu/data/
} 
Table 1. TESS follow-up program transit observations.

\begin{tabular}{|c|c|c|c|c|c|c|c|}
\hline Observatory & $\begin{array}{l}\text { Date } \\
\text { (UTC) }\end{array}$ & Filter & $\begin{array}{c}\text { Exposure } \\
(\mathrm{s})\end{array}$ & $\begin{array}{c}\text { Total } \\
\text { (h) }\end{array}$ & $\begin{array}{c}\text { Aperture } \\
(\mathrm{m})\end{array}$ & $\begin{array}{c}\text { Pixel scale } \\
(\operatorname{arcsec})\end{array}$ & $\begin{array}{c}\text { FOV } \\
(\operatorname{arcmin})\end{array}$ \\
\hline \multicolumn{8}{|l|}{ TOI-776.01 $=$ TOI $-776 c$} \\
\hline MEarth-South, CTIO, Chile & Jul. 1, 2019 & RG715 & 10 & 4.2 & $7 \times 0.4$ & 0.84 & $29 \times 29$ \\
\hline LCOGT, CTIO, Chile & Jul. 1, 2019 & $i^{\prime}$ & 20 & 3.4 & 1.0 & 0.39 & $26.5 \times 26.5$ \\
\hline \multicolumn{8}{|l|}{ TOI-776.02 = TOI-776 b } \\
\hline LCOGT, SAAO, South Africa & Feb. 29, 2020 & $z_{s}$ & 45 & 4.7 & 1.0 & 0.39 & $26.5 \times 26.5$ \\
\hline LCOGT, SSO, Australia & Mar. 17, 2020 & $z_{s}$ & 45 & 4.1 & 1.0 & 0.39 & $26.5 \times 26.5$ \\
\hline PEST, Australia & May 22, 2020 & $R_{\mathrm{C}}$ & 60 & 3.6 & 0.3 & 1.23 & $31 \times 21$ \\
\hline
\end{tabular}

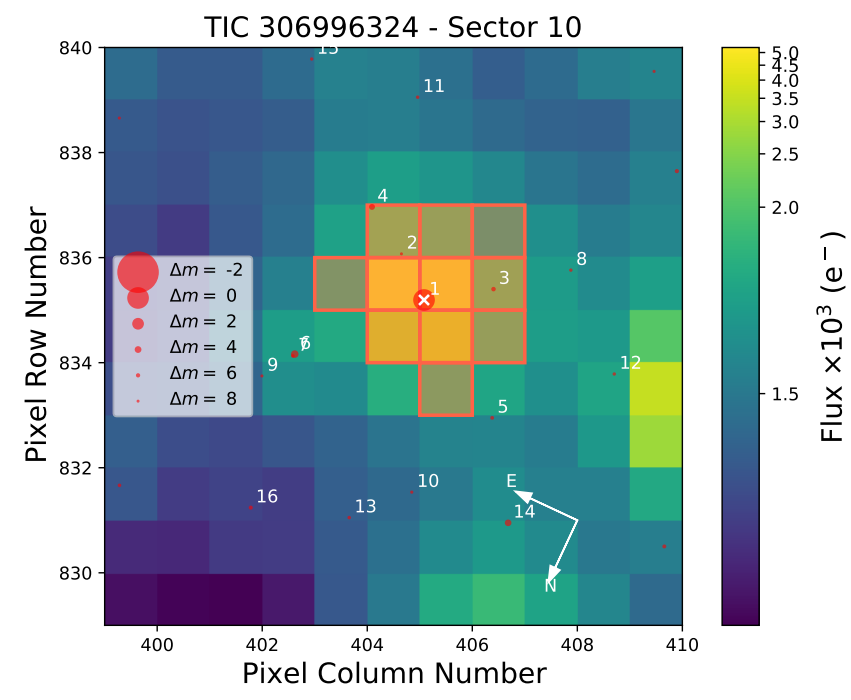

Fig. 1. TESS target pixel file image of LP 961-53 in Sector 10 (created with tpfplotter, Aller et al. 2020). The electron counts are color-coded. The red bordered pixels are used in the simple aperture photometry. The size of the red circles indicates the TESS magnitudes of all nearby stars and LP 961-53 (label \#1 with the " $\times$ "). Positions are corrected for proper motions between the Gaia DR2 epoch (2015.5) and the TESS Sector 10 epoch (2019.2). The TESS pixel scale is approximately $21^{\prime \prime}$.

others. However, the vetting team at the TESS Science Office proposed the possibility that TOI-776.01 could be an EB, where the secondary transit is the primary transit of TOI-776.02 candidate. The ground-based follow-up observations discussed in the next section refuted this scenario and confirmed the two announced candidates as bona-fide planets.

\section{Ground-based observations}

\subsection{Transit follow-up}

We observed the TOI-776 candidates as part of the TESS Followup Observing Program (TFOP) ${ }^{3}$. The goals of these groundbased photometric follow-up observations were to verify that the transits observed by TESS are on target, and to refine the transit ephemeris and depth measurements. We used the TESS Transit Finder, a customized version of the Tapir software package (Jensen 2013), to schedule photometric time-series follow-up observations. We observed two transits of TOI-776.01

\footnotetext{
3 https://tess.mit.edu/followup
}

and three transits of TOI-776.02, as summarized in Table 1 and discussed further below.

\subsubsection{MEarth-South}

A single transit of TOI-776.01 was observed with the $40 \mathrm{~cm}$ MEarth-South telescope array (Irwin et al. 2015) at Cerro Tololo Inter-American Observatory (CTIO), Chile on June 1, 2019. Seven telescopes observed continuously from evening twilight until the target star set below airmass 2 , using an exposure time of $10 \mathrm{~s}$, with all telescopes in focus. The target star was west of the meridian throughout the observation to avoid meridian flips.

Data were reduced following the standard procedures in Irwin et al. (2007) and Berta et al. (2012) with a photometric extraction aperture radius of $r=6$ pix $\left(5^{\prime \prime}\right.$ on sky given the pixel scale of $\left.0 . \mathrm{gix}^{-1}\right)$. The light curve is shown in the lower right of Fig. 3. Due to the large variation in airmass and relatively red target star compared to the available field comparison stars, we found the light curve exhibited a small amount of residual second-order (color-dependent) atmospheric extinction, so the transit model was fit including an extinction term (linear decorrelation against airmass).

\subsubsection{LCOGT}

One transit of TOI-776.01 and two transits of TOI-776.02 were observed with the $1.0 \mathrm{~m}$ telescopes in the Las Cumbres Observatory (LCOGT) telescope network (Brown et al. 2013). The $4096 \times 4096$ pix LCOGT SINISTRO cameras have an image scale of 0.389 pix $^{-1}$, resulting in a $26^{\prime} \times 26^{\prime}$ field of view. The images were calibrated using the standard LCOGT BANZAI pipeline, and photometric data were extracted with AstroImageJ (Collins et al. 2017).

An ingress of TOI-776.01 was observed from the LCOGT node at CTIO on July 1, 2019 in the $i^{\prime}$ filter, simultaneous with the MEarth-South observations mentioned above (Fig. 3, middle right). Transits of TOI-776.02 were observed from the LCOGT nodes at the South African Astronomical Observatory (SAAO) on February 29, 2020 (Fig. 3, middle left) and from the Siding Spring Observatory (SSO) on March 17, 2020, (Fig. 3, lower left). Both observations were made in the $z_{s}$ filter, with the telescopes defocused.

\subsubsection{PEST}

A full transit of TOI-776.02 was observed with the $30 \mathrm{~cm}$ Perth Exoplanet Survey Telescope ${ }^{4}$ (PEST) on May 22, 2020. These

\footnotetext{
4 http://pestobservatory.com/
} 


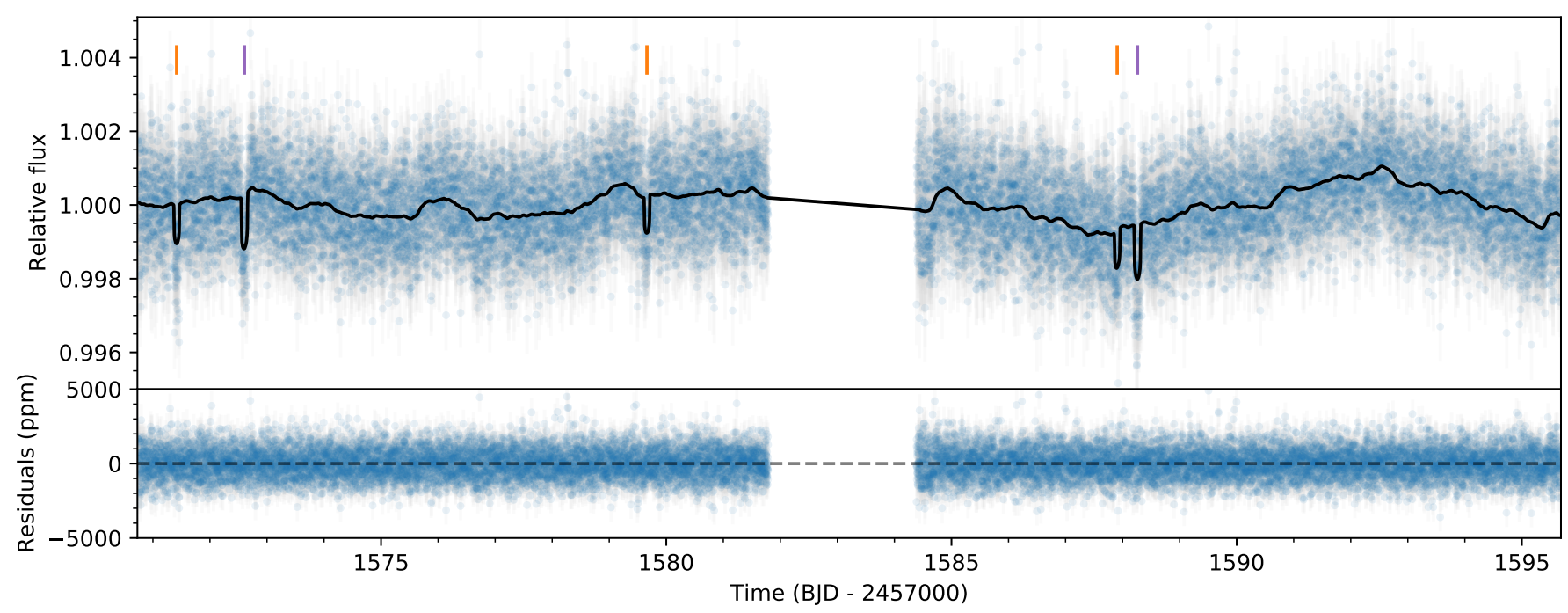

Fig. 2. TESS PDC-corrected SAP transit photometry from SPOC pipeline with the best-fit juliet model (black line; see Sect. 5.2 .1 for details on the modeling). Purple and orange ticks above the light curve mark the transits of the candidates TOI-776.01 (purple) and TOI-732.02 (orange).

data have a scatter that is too large to reliably detect the transit. For this reason, we did not include them in the global fit.

\subsection{Long-term photometric monitoring}

We compiled ground-based, long baseline photometric series from automated surveys. The following public surveys observed TOI-776: the All-Sky Automated Survey for Supernovae (ASAS-SN; Kochanek et al. 2017), All-Sky Automated Survey (ASAS; Pojmanski 2002), Northern Sky Variability Survey (NSVS; Woźniak et al. 2004), and the Catalina surveys (Drake et al. 2014). The telescope location, instrument configurations, and photometric bands of each public survey were summarized in Table 1 of Díez Alonso et al. (2019). All together, the measurements span a period of $15 \mathrm{yr}$.

Additionally, TOI-776 is a candidate of the Super-Wide Angle Search for Planets (SuperWASP; Pollacco et al. 2006). SuperWASP acquired more than 11000 photometric observations, using a broad-band optical filter spanning three consecutive seasons from May to July 2006, January to June 2007, and January to June 2008. In order to detect long-term photometric modulations associated with the stellar rotation, we binned the data into one day intervals, resulting in 201 epochs.

\subsection{High spatial resolution imaging}

The large pixel size of TESS increase the possibility of contamination by nearby sources that are not detected in the seeinglimited photometry or in Gaia DR2. Close companions can dilute the transit depth and thus alter the measured planet radius, or lead to false positives if the companion is itself an EB (e.g., Ciardi et al. 2015). We thus searched for companions by collecting adaptive optics (AO) and speckle images of TOI-776 using $4 \mathrm{~m}$ and $8 \mathrm{~m}$ class telescopes, providing robust limits on the presence of companions and the level of photometric dilution.

\subsubsection{Adaptive optics imaging with Gemini/NIRI and $\mathrm{VLT} / \mathrm{NaCo}$}

On June 15, 2019, TOI-776 was observed using the adaptive optics near-infrared imager (NIRI) mounted on the $8.1 \mathrm{~m}$ Gemini North telescope at Mauna Kea, Hawai'i. We collected a total of $9 \times 1.4 \mathrm{~s}$ images in the $\mathrm{Br} \gamma$ filter centered on $2.166 \mu \mathrm{m}$. We dithered the telescope between exposures, so that the sky background could be constructed from the science frames themselves. After removing bad pixels, flat-fielding, and subtracting the sky background, we aligned the stellar position between frames and co-added the images. The sensitivity of our observations was calculated as a function of radius by injecting fake companions, and scaling their brightness, such that they could be detected at $5 \sigma$. The contrast curve and image are shown in Fig. 4. Only the central $4^{\prime \prime} \times 4^{\prime \prime}$ are shown, but no companions are seen anywhere in the field, which has a field of view of $\sim 13^{\prime \prime} \times 13^{\prime \prime}$.

On July 4, 2019, TOI-776 was observed in $\operatorname{Br} \gamma$ using the NAOS-CONICA AO instrument ( $\mathrm{NaCo}$ ), mounted at the Nasmyth A port of the $8 \mathrm{~m}$ UT1 Very Large Telescope (VLT) in Paranal, Chile. We collected a total of $9 \times 10 \mathrm{~s}$ Br $\gamma$ images. Data were reduced and analyzed using the same procedures as described above for the NIRI data, and no companions were found in the reduced image. The NaCo contrast curve is shown in Fig. 4.

\subsubsection{Speckle imaging with SOAR/HRCam and Gemini/Zorro}

On December 12, 2019, TOI-776 was observed in the $I$ band with a pixel scale of $0.01575^{\prime \prime} \mathrm{pix}^{-1}$ using the HRCam imager, mounted on the $4.1 \mathrm{~m}$ Southern Astrophysical Research (SOAR) telescope at Cerro Tololo Inter-American Observatory, Chile. The data were acquired and reduced following the procedures described in Tokovinin (2018) and Ziegler et al. (2020). The resulting reconstructed image achieved a contrast of $\Delta \mathrm{mag}=7.1$ at a separation of $3^{\prime \prime}$ (see top panel of Fig. 5).

On March 15, 2020, TOI-776 was observed using the Zorro speckle imager (Scott 2019), mounted on the $8.1 \mathrm{~m} \mathrm{Gem-}$ ini South telescope in Cerro Pachón, Chile. Zorro uses high speed electron-multiplying CCDs (EMCCDs) to simultaneously acquire data in two bands centered at 562 and $832 \mathrm{~nm}$. The data were collected and reduced following the procedures described in Howell et al. (2011). The resulting reconstructed image achieved a contrast of $\Delta \mathrm{mag}=7.8$ at a separation of $1^{\prime \prime}$ in the $832 \mathrm{~nm}$ band (see bottom panel of Fig. 5). We note that at the distance of TOI-776, our Zorro speckle images cover a spatial 

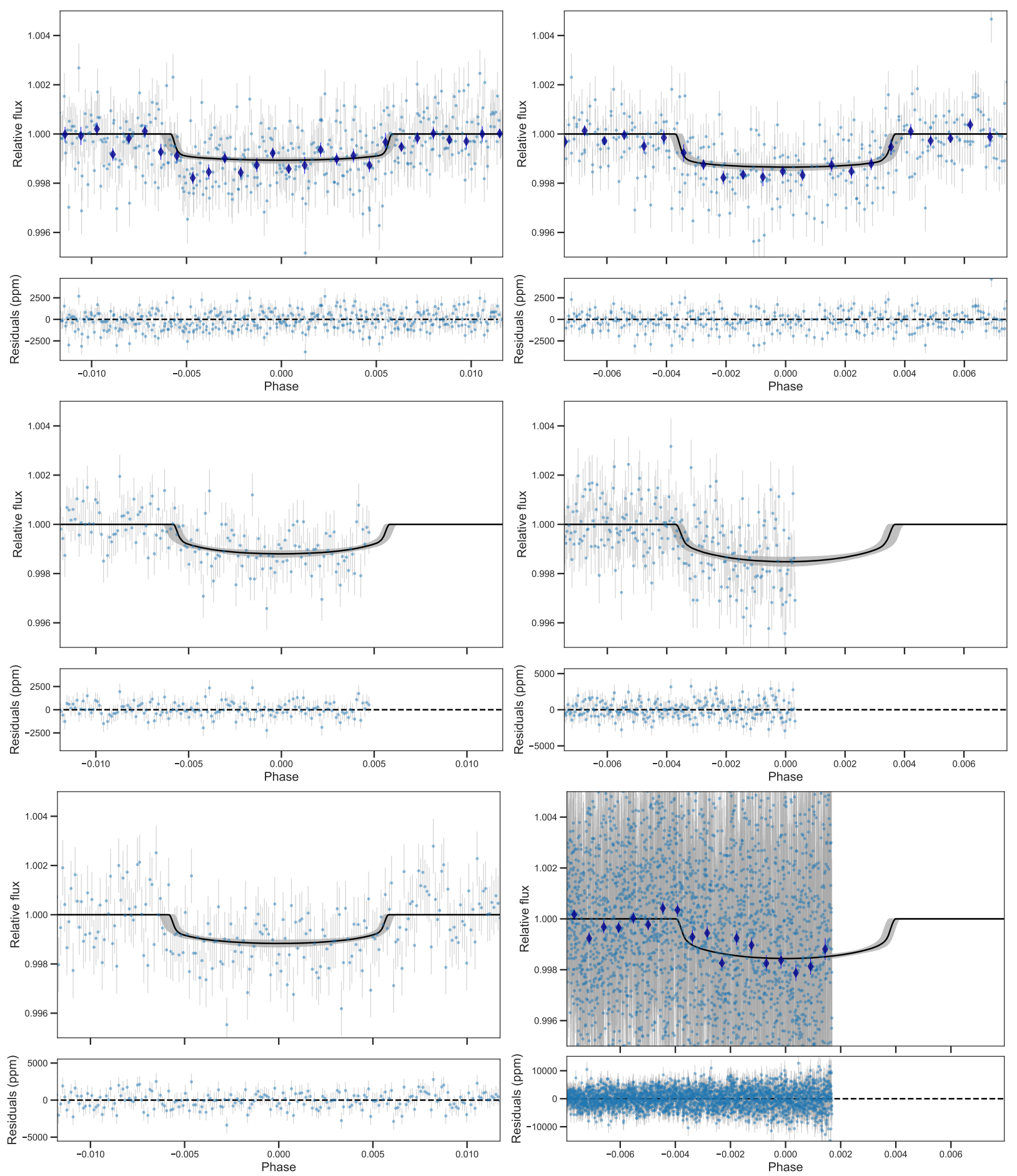

Fig. 3. Phase-folded light curves of TOI-776 b and c. First column: transits of TOI-776 b observed with TESS (top) in Sector 10, LCO-SAAO (middle) on Feb 29, 2020, and LCO-SSO (bottom) on May 22, 2020. Second column: transits of TOI-776 c observed with TESS (top) in Sector 10, and LCO-CTIO (middle) and MEarth-South (bottom) on July 2, 2020. TESS and MEarth-South photometry binned every 10 min are marked with blue diamonds to improve visualization. In all panels, the black lines and shaded areas indicate the detrended best fit model from Sect. 5.2.3 and its $1 \sigma$ confidence interval. Below each panel, the residuals after the subtraction of the median best fit model are represented.

range of $0.46-32$ au around the star with contrasts between 5 and 8 mag.

\subsection{Radial velocity observations}

We obtained 29 high-resolution $(R \approx 115000)$ spectra of TOI776 using the High Accuracy Radial velocity Planet Searcher
(HARPS) spectrograph mounted at the ESO $3.6 \mathrm{~m}$ telescope of La Silla Observatory, Chile (Mayor et al. 2003). The observations were carried out as part of our large observing program 1102.C-0923 (PI: Gandolfi) starting on February 5 and ending on March 23, 2020, when ESO observatories stopped the operations due to the COVID-19 pandemic. One spectrum was acquired under the program 60.A-9709. We used the second 


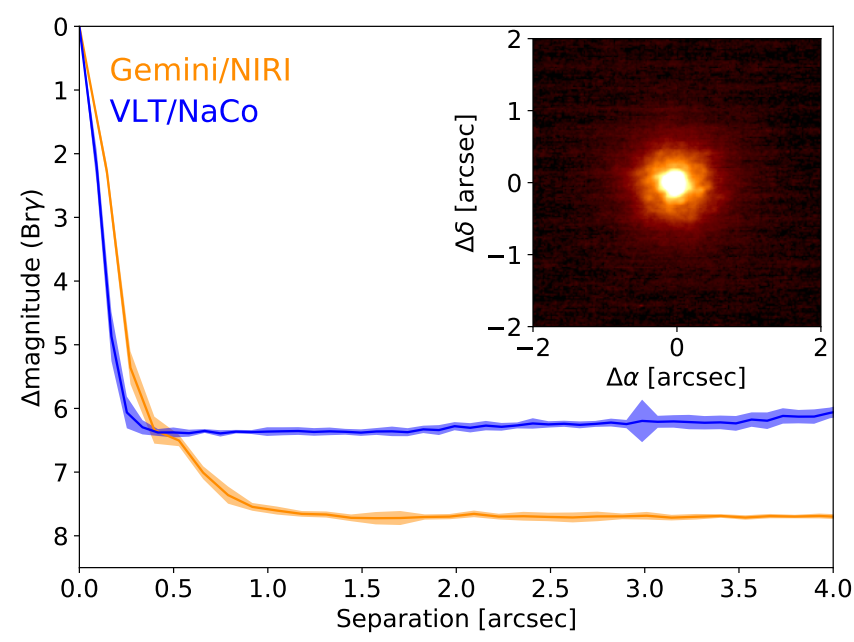

Fig. 4. Contrast curves from NIRI (orange) and $\mathrm{NaCo}$ (blue), and the central $4^{\prime \prime} \times 4^{\prime \prime}$ of the NIRI image (inset). We rule out companions 6 mag fainter than TOI-776 beyond 250 mas, and 7.5 mag fainter beyond 900 mas. The NaCo observations have a slightly tighter inner working angle, while the NIRI observations reach a deeper sensitivity beyond $0.5^{\prime \prime}$
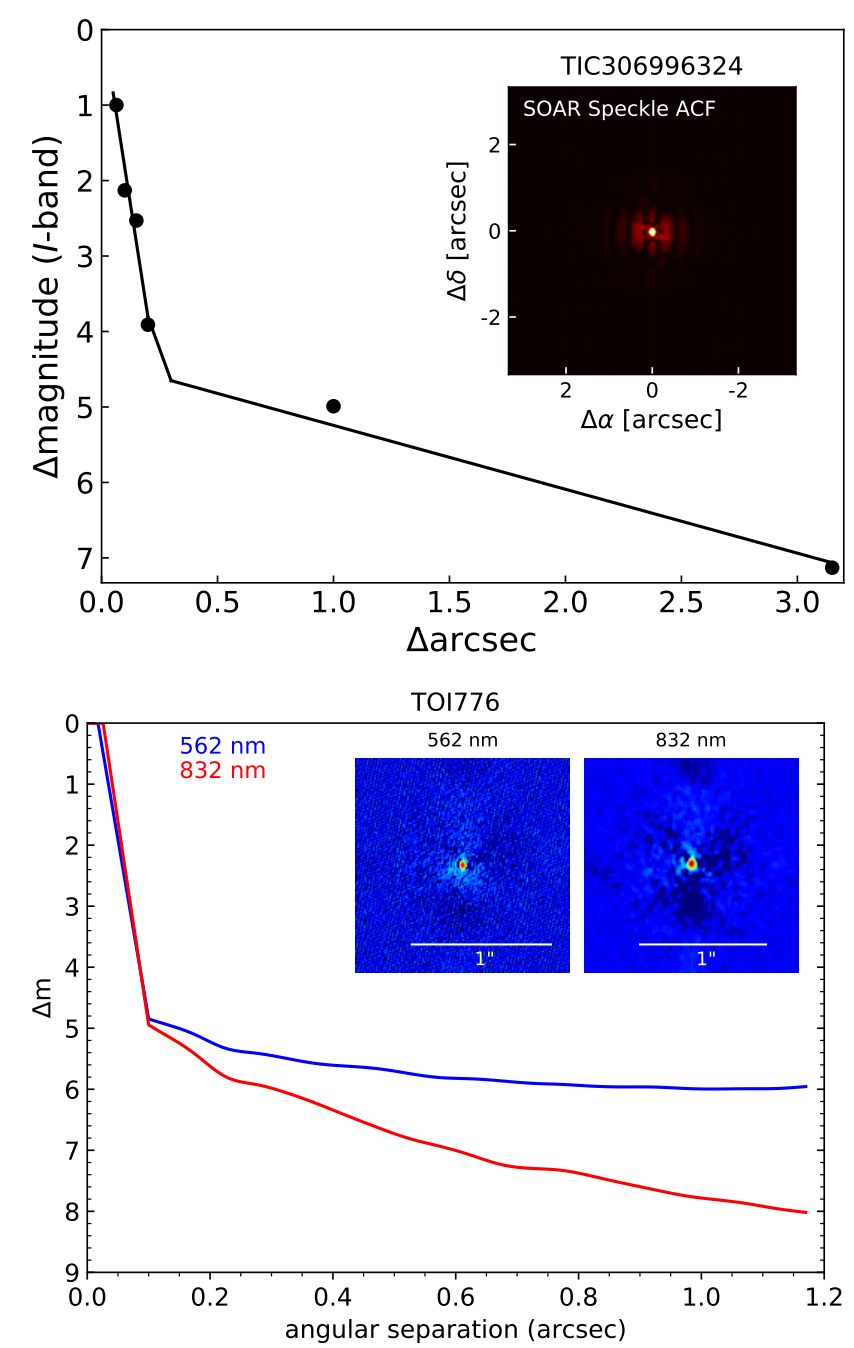

Fig. 5. Top: SOAR contrast curve and $6^{\prime \prime} \times 6^{\prime \prime}$ reconstructed image (inset). Bottom: Gemini/Zorro contrast curves and $1.2^{\prime \prime} \times 1.2^{\prime \prime}$ reconstructed images (inset). fiber of the instrument to monitor the sky background and we reduced the data with the HARPS data reduction software (DRS; Lovis \& Pepe 2007). To compute precise radial velocities and spectral diagnostics, we applied the codes serval (Zechmeister et al. 2018) and TERRA (Anglada-Escudé \& Butler 2012) to the reduced data. Both programs employ a template-matching algorithm that is better suited to derive precise radial velocities for $\mathrm{M}$ dwarfs if compared to the cross-correlation function (CCF) technique implemented in the DRS. In the CCF technique, the line lists of $\mathrm{M}$ dwarfs used to define the binary mask are incomplete and they thus produce a $\mathrm{CCF}$ that is often a poor match for cool stars. The RVs have a median internal uncertainty of $1.5 \mathrm{~m} \mathrm{~s}^{-1}\left(1.5 \mathrm{~m} \mathrm{~s}^{-1}\right)$ and a root mean square of $5.2 \mathrm{~m} \mathrm{~s}^{-1}$ $\left(3.5 \mathrm{~m} \mathrm{~s}^{-1}\right)$ around the mean value for the serval (TERRA) extractions, respectively. We report in Tables B.1 and B.2 the HARPS measurements; the extracted RVs and the associated uncertainties; $\mathrm{Na}$ I D, Na II D and $\mathrm{H} \alpha$ line indices from both programs together with the chromatic index (CRX) and differential line width (dLW) computed by serval; and the Mount Wilson S-index computed by TERRA.

\section{Stellar properties}

\subsection{Stellar parameters}

The star TOI-776 belongs to the Catalog Of Nearby Cool HostStars for Habitable ExopLanets and Life (CONCH-SHELL) compiled by Gaidos et al. (2014). For an all-sky sample of approximately $3000 \mathrm{M}$ - or late K-type stars, the authors provide spectroscopically determined values of the spectral type, effective temperature, and metallicity; which, combined with empirical relations for cool stars, make it possible to estimate stellar radius, luminosity, and mass. In particular, they measure that TOI-776 is a relatively inactive M1 V dwarf star with the stellar properties shown in Table 2.

We carried out an independent analysis to improve the photospheric and fundamental parameters of TOI-776. We used SpecMatch-Emp (Yee et al. 2017) to empirically estimate the effective temperature, metallicity, and stellar radius by comparing the co-added HARPS high-resolution spectrum with a spectroscopic library of well-characterized stars. The results of this analysis are in agreement with the values of Gaidos et al. (2014) within the errors. Then, we derived the stellar radius and luminosity combining Gaia $G, G_{\mathrm{BP}}, G_{\mathrm{RP}}$ photometry and 2MASS $J, H, K_{\mathrm{S}}$ magnitudes with the spectroscopic parameters from the SpecMatch-Emp analysis and the Gaia parallax. We corrected the Gaia $G$ photometry for the magnitude-dependent offset using Eq. (3) from Casagrande \& VandenBerg (2018) and adopted a minimum uncertainty of $0.01 \mathrm{mag}$ for the Gaia magnitudes to account for additional systematic uncertainties. We added 0.06 mas to the nominal Gaia parallax to account for the systematic offset found by Stassun \& Torres (2018); Riess et al. (2018), and Zinn et al. (2019). Our best estimate of the stellar radius is consistent with the value from Gaidos et al. (2014) and in agreement with each of the radius estimates obtained independently using only one of the magnitudes. Finally, we computed the mass using the mass-radius relations for $\mathrm{M}$ dwarfs from Schweitzer et al. (2019).

We also applied the methods of Reddy et al. (2006) to Gaia DR2 astrometry for TOI-776 to compute galactic $U, V, W$ velocities in the local standard of rest and the probabilities of kinematic membership in galactic stellar populations. We found that TOI-776 has a $96.3 \%$ probability of belonging to the thin disk population, which is in excellent agreement with the galactic 
Table 2. Stellar parameters of TOI-776.

\begin{tabular}{|c|c|c|}
\hline$\underline{\text { Parameter }}$ & Value & Reference \\
\hline \multicolumn{3}{|c|}{ Name and identifiers } \\
\hline Name & LP 961-53 & Luyten (1974) \\
\hline TOI & 776 & TESS Science Office \\
\hline TIC & 306996324 & Stassun et al. (2018) \\
\hline \multicolumn{3}{|c|}{ Coordinates and spectral type } \\
\hline$\alpha$ & $11: 54: 18.39$ & Gaia DR2 \\
\hline$\delta$ & $-37: 33: 09.8$ & Gaia DR2 \\
\hline $\mathrm{SpT}$ & M1 V & Gaidos et al. (2014) \\
\hline \multicolumn{3}{|c|}{ Magnitudes } \\
\hline$V(\operatorname{mag})$ & $11.54 \pm 0.04$ & UCAC4 \\
\hline$g$ (mag) & $12.35 \pm 0.12$ & UCAC4 \\
\hline$G$ (mag) & $10.7409 \pm 0.0005$ & Gaia DR2 \\
\hline$r(\mathrm{mag})$ & $10.92 \pm 0.03$ & UCAC4 \\
\hline$i$ (mag) & $10.05 \pm 0.09$ & UCAC4 \\
\hline$J$ (mag) & $8.483 \pm 0.018$ & 2MASS \\
\hline$H$ (mag) & $7.877 \pm 0.040$ & 2MASS \\
\hline$K_{\mathrm{s}}(\mathrm{mag})$ & $7.615 \pm 0.020$ & 2MASS \\
\hline \multicolumn{3}{|c|}{ Parallax and kinematics } \\
\hline$\pi$ (mas) & $36.78 \pm 0.04$ & Gaia DR2 \\
\hline$d(\mathrm{pc})$ & $27.19 \pm 0.03$ & Gaia DR2 \\
\hline$\mu_{\alpha} \cos \delta\left(\operatorname{mas~yr}^{-1}\right)$ & $+251.112 \pm 0.051$ & Gaia DR2 \\
\hline$\mu_{\delta}\left(\operatorname{mas~yr}^{-1}\right)$ & $-145.059 \pm 0.083$ & Gaia DR2 \\
\hline$V_{\mathrm{r}}\left(\mathrm{km} \mathrm{s}^{-1}\right)$ & $49.34 \pm 0.22$ & Gaia DR2 \\
\hline$U\left(\mathrm{~km} \mathrm{~s}^{-1}\right)$ & $60.71 \pm 0.08$ & This work $^{(a)}$ \\
\hline$V\left(\mathrm{~km} \mathrm{~s}^{-1}\right)$ & $-28.27 \pm 0.17$ & This work ${ }^{(a)}$ \\
\hline$W\left(\mathrm{~km} \mathrm{~s}^{-1}\right)$ & $18.73 \pm 0.09$ & This work $^{(a)}$ \\
\hline \multicolumn{3}{|c|}{ Photospheric parameters } \\
\hline$T_{\text {eff }}(\mathrm{K})$ & $3709 \pm 70$ & This work \\
\hline & $3766 \pm 100$ & Gaidos et al. (2014) \\
\hline $\log g$ & $4.727 \pm 0.025$ & This work \\
\hline$(\mathrm{Fe} / \mathrm{H})$ & $-0.20 \pm 0.12$ & This work \\
\hline \multicolumn{3}{|c|}{ Physical parameters } \\
\hline$R\left(R_{\odot}\right)$ & $0.538_{-0.024}^{+0.024}$ & This work \\
\hline \multirow{3}{*}{$L\left(L_{\odot}\right)$} & $0.53 \pm 0.05$ & Gaidos et al. (2014) \\
\hline & $0.049 \pm 0.002$ & This work \\
\hline & $0.050 \pm 0.013$ & Gaidos et al. (2014) \\
\hline \multirow[t]{2}{*}{$M\left(M_{\odot}\right)$} & $0.544_{-0.028}^{+0.028}$ & This work \\
\hline & $0.56 \pm 0.07$ & Gaidos et al. (2014) \\
\hline Age (Gyr) & $7.8_{-6.3}^{+3.9}$ & This work \\
\hline
\end{tabular}

Notes. ${ }^{(a)}$ Computed in the local standard of rest.

References. Gaia DR2: Gaia Collaboration (2018); UCAC4: Zacharias et al. (2013); 2MASS: Skrutskie et al. (2006).

population probabilities for this star in the recent catalog of Carrillo et al. (2020). Additionally, using the code isochrones (Morton 2015), we estimated the age of TOI-776 to be loosely constrained between 2 and 10 Gyr. From the metallicity, age, and kinematics given in Table 2, we can conclude that TOI-776 is a relatively old member of the galactic thin disk population.

\subsection{Stellar rotation period}

To determine the rotational period of the star, we used the publicly available photometric data for TOI-776. Using juliet

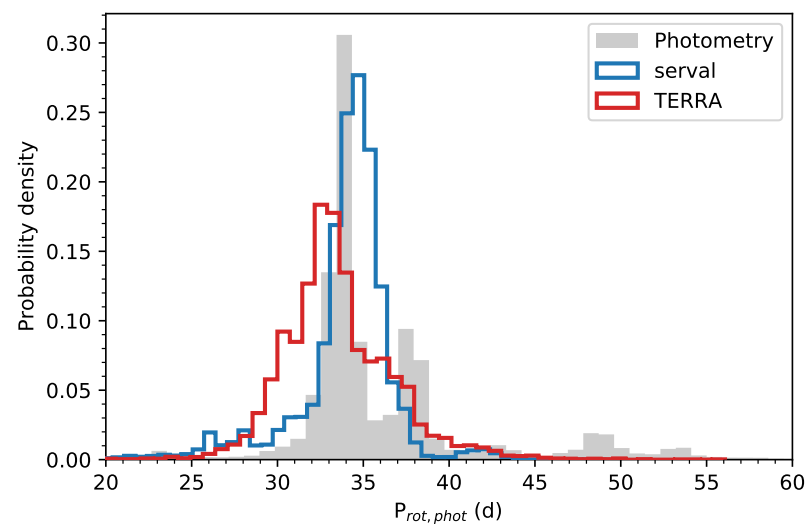

Fig. 6. Probability density of the samples of the $P_{\text {rot }}$ parameter from the GP fit of the ground-based, long-term photometric monitoring (gray) from Sect. 4.2 and of the period of the additional sinusoidal signal from the RV fit from Sect. 5.2.2 using serval (blue) or TERRA (red) reductions.

(see more details about the algorithm in Sect. 5.2), we modeled the ASAS-SN, ASAS, NSVS, Catalina, and daily binned SuperWASP data with Gaussian processes (GPs). In particular, we adopted the quasi-periodic GP kernel introduced in Foreman-Mackey et al. (2017) of the form

$k_{i, j}(\tau)=\frac{B}{2+C} e^{-\tau / L}\left[\cos \left(\frac{2 \pi \tau}{P_{\text {rot }}}\right)+(1+C)\right]$,

where $\tau=\left|t_{i}-t_{j}\right|$ is the time-lag, $B$ and $C$ define the amplitude of the GP, $L$ is a timescale for the amplitude-modulation of the GP, and $P_{\text {rot }}$ is the rotational period of the modulations. As in Luque et al. (2019), we considered each of the five data sets to have different values of $B$ and $C$, in order to account for the possibility that different bands could have different GP amplitudes, while we imposed the timescale of the modulation and the rotational period as common parameters for all the data sets. In addition, we fit an extra jitter term for each photometric time series. We considered wide uninformative priors for the jitter, $B, C, L$, and a uniform rotation period prior between 10 and $100 \mathrm{~d}$.

Figure 6 shows the posterior samples of the GP hyperparameter $P_{\text {rot }}$ after fitting all the long-term monitoring ground-based photometry. The distribution is bimodal with peaks at $33 \pm 1 \mathrm{~d}$ and $38 \pm 1 \mathrm{~d}$, where the samples from the first peak have the highest likelihood. From this we can estimate that the stellar rotation of TOI-776 is between 30 and $40 \mathrm{~d}$ over the course of $15 \mathrm{yr}$. The $38 \mathrm{~d}$ peak may be an alias of the true $33 \mathrm{~d}$ rotation due to $1 \mathrm{yr}$ window function in the photometry. Alternatively, the bimodal distribution of the $P_{\text {rot }}$ can be explained as a consequence of the stellar differential rotation coupled with the activity cycle (Rüdiger et al. 2014; Küker et al. 2019). For early M dwarfs with rotational periods similar to TOI-776, the expected dynamo cycle time is between 3 and 6 yr (Küker et al. 2019), thus detectable in our data. Additionally, assuming that this star is a solar-like rotator, the rotational velocity of the star decreases as the latitude increases. The two peaks correspond to two different groups of activity features, a bigger one, closer to the equator, which generates the first peak of the posterior distribution, and a smaller one, at a higher latitude, which produces the second peak. The opposite situation, with an antisolar-like rotator, is less likely, considering that TOI-776 is an adult star, still belonging to the main sequence. 


\section{Analysis}

\subsection{Frequency analysis of the HARPS data}

We performed a frequency analysis of the HARPS serval/TERRA extracted measurements to search for the Doppler reflex motion induced by the two transiting planets discovered in the TESS light curve and to unveil the presence of additional signals associated with the star and/or other orbiting planets. Figure 7 shows the generalized Lomb Scargle (GLS; Zechmeister et al. 2009) periodograms of the HARPS RVs and activity indicators extracted with serval (blue lines) and with TERRA (red lines). The horizontal dashed lines mark the GLS powers corresponding to the 0.1 , 1 , and $5 \%$ false alarm probability $(\mathrm{FAP})^{5}$. The vertical dashed lines mark the orbital frequencies of the two transiting planets detected in the TESS light curve $\left(f_{\mathrm{b}}=0.121 \mathrm{~d}^{-1}\right.$ and $\left.f_{\mathrm{c}}=0.064 \mathrm{~d}^{-1}\right)$ and the stellar signal at $\sim 0.03 \mathrm{~d}^{-1}$ (see below).

The upper panel of Fig. 7 displays the GLS periodogram of the HARPS RVs in the $0-0.42 \mathrm{~d}^{-1}$ frequency range. The highest peak is found at $0.055 \mathrm{~d}^{-1}(\mathrm{FAP} \approx 0.3 \%)$, which is close to the orbital frequency of TOI-776 $\mathrm{c}\left(f_{\mathrm{c}}=0.064 \mathrm{~d}^{-1}\right)$. Taking into account our frequency resolution ${ }^{6}$ of $0.021 \mathrm{~d}^{-1}$, the two frequencies are indistinguishable. This suggests that the highest peak seen in the periodogram of the HARPS RVs is the stellar reflex motion induced by the outer transiting planet TOI-776 c. The second highest peak is found at $0.129 \mathrm{~d}^{-1}$ (Fig. 7, upper panel), which is close to the orbital frequency of TOI-776 b. However, this signal is an alias of the signal at $0.055 \mathrm{~d}^{-1}$. The periodogram of the window function indeed shows a peak at $0.074 \mathrm{~d}^{-1}$ (highlighted with an arrow in the bottom panel of Fig. 7), which is equal to the frequency spacing between the two highest peaks seen in the periodogram of the HARPS RVs.

We used the code pyaneti (Barragán et al. 2019, see also Sect. 5.2.3) to subtract the Doppler signal of TOI-776 c from the HARPS RVs. We assumed a circular model (see also Sect. 5.2.2), fixing period and time of first transit to the TESS ephemeris, while allowing for the systemic velocity and RV semi-amplitude to vary. The periodogram of the RV residuals shows a broad peak centered around $\sim 0.04 \mathrm{~d}^{-1}$ with a FAP of about $10 \%$. Although the Doppler signal is not significant, the GLS periodograms of the CRX, dLW, $\mathrm{H} \alpha$, and S-index activity indicators show also peaks at $\sim 0.04 \mathrm{~d}^{-1}$, suggesting that this signal is caused by the presence of active regions appearing and disappearing from the visible stellar disk as the star rotates around its axis. It is worth noting that the peak at $0.130 \mathrm{~d}^{-1}$ is not observed in the GLS periodogram of the RV residuals, corroborating the interpretation that this peak is an alias of the dominant frequency detected in the periodogram of the HARPS data.

We removed the Doppler reflex motion of TOI-776 $\mathrm{c}$ and the activity-induced RV signal by jointly modeling the HARPS measurements with a circular Keplerian orbit and a sine curve. For TOI-776 c, we followed the same procedure described in the previous paragraph. For the stellar signal, we fit for the phase, amplitude, and frequency. The latter was allowed to vary within a wide uniform prior centered around $0.04 \mathrm{~d}^{-1}$. The GLS

5 Following the bootstrap method described, for example, in Murdoch et al. (1993) and Hatzes (2016), we estimated the FAP by computing the GLS periodogram of $10^{6}$ time series obtained by randomly shuffling the measurements and their uncertainties, while keeping the time stamps fixed.

6 The frequency resolution is defined as the inverse of the time baseline. The baseline of our HARPS observations is about 47 days, corresponding to a frequency resolution of about $1 / 47=0.021 \mathrm{~d}^{-1}$.
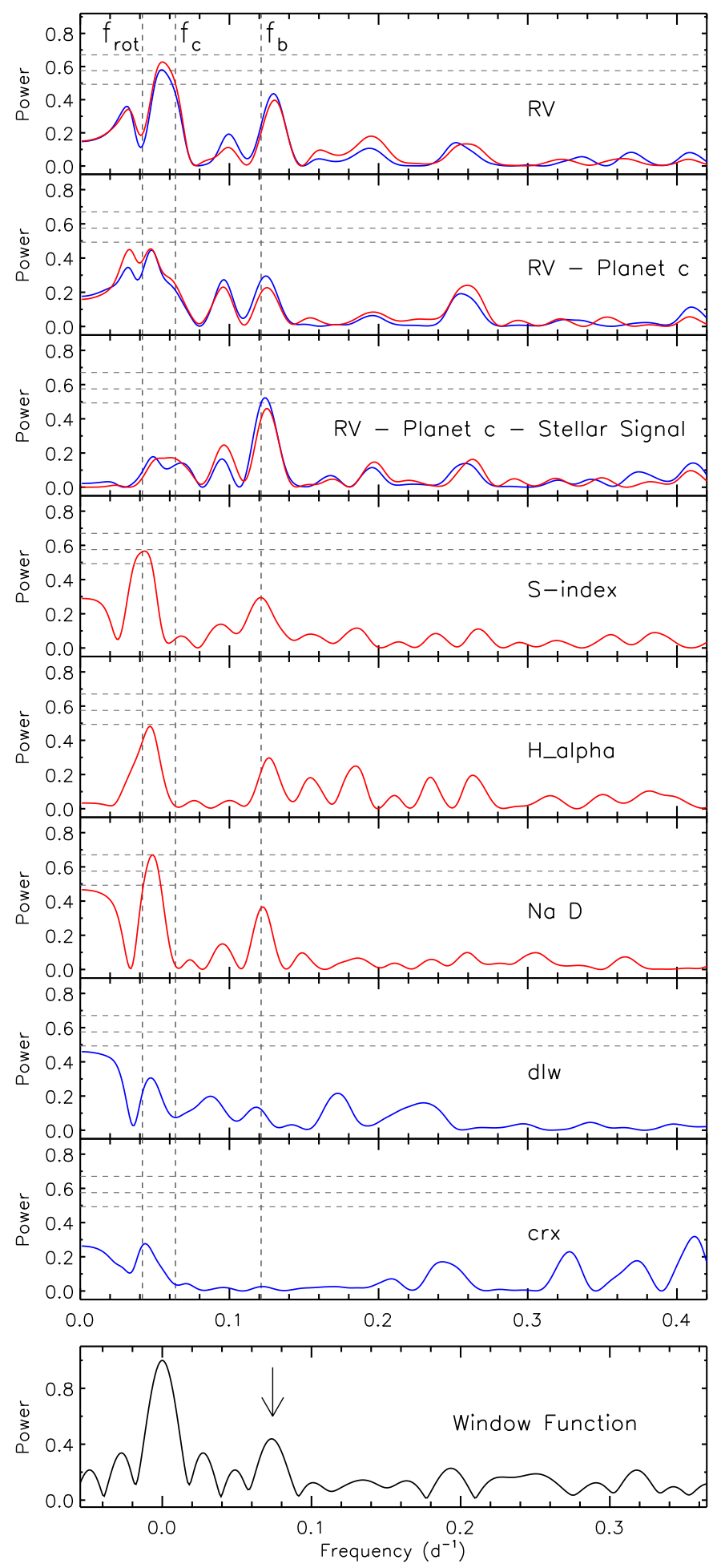

Fig. 7. Generalized Lomb-Scargle periodograms of the HARPS RVs and spectral activity indicators from serval (blue) and TERRA (red). The horizontal dashed lines mark, from bottom to top, the 5, 1, and $0.1 \%$ FAP levels, respectively. The vertical dashed lines mark the orbital frequencies of the two transiting planets $\left(f_{\mathrm{b}}=0.121 \mathrm{~d}^{-1}\right.$ and $\left.f_{\mathrm{c}}=0.064 \mathrm{~d}^{-1}\right)$ and of the stellar signal at $\sim 0.03 \mathrm{~d}^{-1}$. Upper panel: HARPS RVs. Second panel: RV residuals following the subtraction of the signal of TOI-776 $\mathrm{c}$. Third panel: RV residuals following the subtraction of the reflex motion of TOI-776 $\mathrm{c}$ and of the activity-induced stellar signal. Fourth panel: S-index. Fifth panel: $\mathrm{H} \alpha$ line. Sixth panel: Na D lines. Seventh panel: differential line width (dLW). Eighth panel: chromatic index (CRX). Bottom panel: window function. The arrow in the bottom panel indicates the peak at $0.07 \mathrm{~d}^{-1}$ referred to in the discussion of Sect. 5.1. 
periodogram of the RV residuals displays a peak at $0.125 \mathrm{~d}^{-1}$ $(\mathrm{FAP} \approx 11 \%$ ), which is very close to the frequency of the inner transiting planet TOI-776 $\mathrm{b}\left(f_{\mathrm{b}}=0.121 \mathrm{~d}^{-1}\right)$. We note that the activity indicators also show peaks close to the orbital frequency of TOI-776 b. Yet, those peaks are separated by $0.074 \mathrm{~d}^{-1}$ from the stellar signal at $\sim 0.04 \mathrm{~d}^{-1}$. As such they are very likely aliases of the latter.

\subsection{Modeling results}

In this section, we describe our use of juliet (Espinoza et al. 2019) to model the photometric and Doppler data, both separately and jointly. The algorithm is built on several publicly available tools that model transits (batman, Kreidberg 2015), RVs (radvel, Fulton et al. 2018), and GPs (george, Ambikasaran et al. 2015; celerite, Foreman-Mackey et al. 2017).

\subsubsection{Photometry}

First, to constrain the properties of the transiting planets and use them for further analyses, we modeled the TESS, LCO, and MEarth photometry with juliet. We adopted a quadratic limb darkening law for TESS, since Espinoza \& Jordán (2015) showed it was equally appropriate for space-based missions. The limb darkening parameters were then parameterized with a uniform sampling prior $\left(q_{1}, q_{2}\right)$, introduced by Kipping (2013). For LCO and MEarth transits, we used a more simple linear limb darkening law, because the lower data precision with respect to TESS prevents us from adopting a more complex law. Additionally, we followed the parameterization introduced in Espinoza (2018). In particular, for each transiting planet, rather than fitting for the planet-to-star radius ratio $p=R_{\mathrm{p}} / R_{*}$ and the impact parameter of the orbit $b$, we sampled from the uniform priors assigned to two parameters, $r_{1}$ and $r_{2}$, which are connected to $\mathrm{p}$ and $\mathrm{b}$ with Eqs. (1)-(4) in Espinoza (2018). $r_{1}$ and $r_{2}$ were shown in Espinoza (2018) to guarantee a full exploration of the physically plausible values in the $(\mathrm{p}, \mathrm{b})$ plane. We also assumed circular orbits and fixed the TESS dilution factor to 1, based on our analysis from Sect. 3.3. Finally, we added a jitter term $\sigma$ in quadrature to the TESS, LCO, and MEarth photometric uncertainties. The details of the priors and the description for each parameter are presented in Table A.1.

To account for the time-correlated noise in the light curve in Fig. 2, even using the PDC-corrected SAP, we modeled the TESS photometry with the exponential GP kernel

$k_{i, j}=\sigma_{\mathrm{GP}, \mathrm{TESS}}^{2} \exp \left(-\left|t_{i}-t_{j}\right| / T_{\mathrm{GP}, \mathrm{TESS}}\right)$,

where $T_{\mathrm{GP}, \mathrm{TESS}}$ is a characteristic timescale, and $\sigma_{\mathrm{GP}, \mathrm{TESS}}$ is the amplitude of this GP modulation. For the LCO photometry, on the other hand, we used a linear model to detrend the data from airmass correlations.

Our photometry-only analysis significantly increases the precision of the planet parameters with respect to the TESS DVR. The uncertainties in the period decreases by two orders of magnitudes, which eases up future ground- and space-based follow-up efforts. The radii of the planets are determined to a precision better than 5\%. Finally, we searched for an additional planet in the system by modeling a three-planet fit with the same priors as in Table A.1 for the transiting planets, and by varying the period and mid-transit time of the third hypothetical planet. Our result notably excludes the presence of any additional transits in the light curve $\left(\Delta \ln Z=\ln Z_{2 \mathrm{pl}}-\ln Z_{3 \mathrm{pl}}>7\right)$.
Table 3. Model comparison of RV-only fits with juliet.

\begin{tabular}{lllcc}
\hline \hline Model & Prior $P_{\text {planet }}$ & $\mathrm{GP}$ & $\ln Z_{\text {serval }}$ & $\ln Z_{\text {TERRA }}$ \\
\hline $0 \mathrm{pl}$ & $\ldots$ & $\ldots$ & -84.3 & -85.4 \\
$2 \mathrm{pl}$ & $\mathcal{N}_{\mathrm{b}}\left(8.24,0.05^{2}\right)$ & $\ldots$ & -81.3 & -81.8 \\
& $\mathcal{N}_{\mathrm{c}}\left(15.65,0.05^{2}\right)$ & & & \\
$2 \mathrm{pl}+\mathrm{GP} 1$ & $\mathcal{N}_{\mathrm{b}}\left(8.24,0.05^{2}\right)$ & $\mathrm{EXP}^{(a)}$ & -80.7 & -81.7 \\
& $\mathcal{N}_{\mathrm{c}}\left(15.65,0.05^{2}\right)$ & & & \\
2 pl+GP2 & $\mathcal{N}_{\mathrm{b}}\left(8.24,0.05^{2}\right)$ & $\mathrm{ESS}^{(b)}$ & -80.6 & -81.8 \\
& $\mathcal{N}_{\mathrm{c}}\left(15.65,0.05^{2}\right)$ & & & \\
2pl+sinusoid & $\mathcal{N}_{\mathrm{b}}\left(8.24,0.05^{2}\right)$ & $\ldots$ & $-\mathbf{7 8 . 9}$ & -79.4 \\
& $\mathcal{N}_{\mathrm{c}}\left(15.65,0.05^{2}\right)$ & & & \\
& $\mathcal{N}_{\mathrm{d}}\left(35.0,10.0^{2}\right)$ & & & \\
\hline
\end{tabular}

Notes. The prior label $\mathcal{N}$ represents a normal distribution. The final model used for the joint fit is marked in boldface (see Sect. 5.2.2 for details about the selection of the final model). ${ }^{(a)}$ Simple exponential kernel (EXP) of the form $k_{i, j}=\sigma_{\mathrm{GP}, \mathrm{RV}}^{2} \exp \left(-\left|t_{i}-t_{j}\right| / T_{\mathrm{GP}, \mathrm{RV}}\right)$. ${ }^{(b)}$ Exponential-sine-squared kernel (ESS) of the form $k_{i, j}=$ $\sigma_{\mathrm{GP}, \mathrm{RV}}^{2} \exp \left(-\alpha_{\mathrm{GP}, \mathrm{RV}}\left(t_{i}-t_{j}\right)^{2}-\Gamma_{\mathrm{GP}, \mathrm{RV}} \sin ^{2}\left[\frac{\pi\left|t_{i}-t_{j}\right|}{P_{\mathrm{rot} ; \mathrm{GP}, \mathrm{RV}}}\right]\right)$ with a uniform prior in $P_{\text {rot } ; \mathrm{GP}, \mathrm{RV}}$ ranging from 5 to $50 \mathrm{~d}$.

\subsection{2. $\mathrm{RV}$}

Even though the results of the RV's extraction slightly change depending on whether we used serval or TERRA, the GLS analyses in both cases show evidence of a stellar signal together with the RV trends associated with the transiting planets. To adequately describe the data, we considered several RV-only models and carried out a model comparison scheme as in Luque et al. (2019). We used juliet, a code that efficiently computes the Bayesian log-evidence of each tested model and explores the parameter space using the importance nested sampling included in MultiNest (Feroz et al. 2009) via the PyMultiNest package (Buchner et al. 2014). As discussed in Nelson et al. (2020), this method outperforms other samplers in robustly choosing the best model for those with three or fewer planets. We considered a model to be moderately favored over another if the difference in its Bayesian log-evidence $\Delta \ln Z$ is greater than two, while it is strongly favored if it is greater than five (Trotta 2008). If $\Delta \ln Z \lesssim 2$, then the models are indistinguishable. In this case, the model with fewer degrees of freedom would be chosen.

Due to the sampling and the scarce number of RV measurements, if we model the eccentricity with a wide, uninformative prior, we derive nonphysically high eccentricities for both planets that would make the system unstable in fewer than one hundred orbits. The eccentricity of systems with multiple transiting planets is low but not necessarily zero (Van Eylen \& Albrecht 2015; Xie et al. 2016; Hadden \& Lithwick 2017). Therefore, instead of assuming circular orbits, we placed a prior on the orbital eccentricity of a beta distribution with $\alpha=1.52$ and $\beta=29$ following Van Eylen et al. (2019). Table 3 summarizes the results of our analysis on both serval- or TERRA-extracted RVs. As seen in Table 3, including the two transiting planets in the model is favored over the fiducial model $(0 \mathrm{pl})$. On the other hand, we tested different types of two-planet models. First, we considered just the two transiting planets $(2 \mathrm{pl})$, without accounting for additional noise sources. Then, we accounted for the stellar noise, modeling it in three different ways: with an exponential GP kernel (2pl+GP1), with an exponential sine-squared 
GP kernel (2pl+GP2) and with a simple sinusoid (2pl+sinusoid). All the tested two-planet models are statistically indistinguishable, with their Bayesian $\log$-evidences within $\Delta \ln Z<2$.

However, for both serval and TERRA-extracted RVs, the nominal best model accounts for two circular orbits and an additional sinusoidal curve, whose period is equal to the stellar period of rotation we estimated through the long-term groundbased photometric data. For this test, we imposed a normal prior on $P_{\text {rot }}$, with a wide standard deviation (10). We additionally tried wide, uninformative priors for the period of the sinusoidal signal, and we retrieved the same posterior distributions and log evidence (Fig. 6) as for the test with a Gaussian prior. With the RV analysis, we estimated a stellar period of rotation $P_{\text {rot }}=34.4_{-2.0}^{+1.4} \mathrm{~d}$, consistent with the rotational period estimated from the ground-based long-term photometry in Sect. 4.2. Additionally, all models presented in Table 3 derive the same RV semi-amplitude for TOI-776 b and TOI-776 c, well within their $1 \sigma$ uncertainties. This proves the robustness of the mass determination for the transiting planets, independently of the stellar noise distribution.

Leveraging the prior information on the stellar rotation from photometry discussed in Sect. 4.2 with the presence of a significant periodicity in the RV residuals of a two-planet model (Fig. 7b), we decided to choose the 2 pl+sinusoid as our final model for the joint fit. With respect to the RV extraction, we preferred to use the serval extracted RVs in the final joint fit due to their nominal highest log-evidence and lower jitter compared to TERRA.

\subsubsection{Joint fit}

We performed a joint fit using juliet of the TESS, LCO, and MEarth photometry and HARPS serval extracted RVs, using the 2pl+sinusoid model we selected after the RV-only analysis in Sect. 5.2.2. Tables A.1 and 4 show the priors and posteriors of all the fit parameters, respectively. Figure C.1 shows a corner plot of the orbital parameters of planets $b$ and c. The data, residuals, and joint fit of the preferred model are shown in Figs. 3 and 8 for the photometry and the RVs, respectively. Table 5 lists the transit and physical parameters derived using the stellar parameters in Table 2.

As a sanity check, we performed an independent joint analysis of the transit photometry and Doppler measurements using the pyaneti code (Barragán et al. 2019), which estimates the parameters of planetary systems in a Bayesian framework, combined with an MCMC sampling. We imposed uniform priors for all the fit parameters. Following Winn (2010), we sampled for the mean stellar density $\rho_{\star}$ and recovered the scaled semi-major axis $\left(R_{\mathrm{p}} / R_{\star}\right)$ for each planet using Kepler's third law. We found that the modeling of the transit light curves provides a mean stellar density of $\rho_{\star}=5203_{-1228}^{+1782} \mathrm{~kg} \mathrm{~m}^{-3}$, which agrees with the density of $4834_{-559}^{+651} \mathrm{~kg} \mathrm{~m}^{-3}$ derived from the stellar mass and radius presented in Sect. 4. As for the remaining parameters, the analysis provides parameter estimates consistent with those derived with juliet, thus corroborating our results.

\section{Results and discussion}

The TOI-776 system consists of two transiting planets. The inner planet, TOI-776 b, has a period of $8.25 \mathrm{~d}$, a radius of $1.85 \pm 0.13 R_{\oplus}$, a mass of $4.0 \pm 0.9 M_{\oplus}$, and a bulk density of $3.4_{-0.9}^{+1.1} \mathrm{~g} \mathrm{~cm}^{-3}$. The outer planet, TOI-776 $\mathrm{c}$, has a period of $15.66 \mathrm{~d}$, a radius of $2.02 \pm 0.14 R_{\oplus}$, a mass of $5.3 \pm 1.8 M_{\oplus}$, and a
Table 4. Median and $68 \%$ credibility intervals of the posterior distributions for each fit parameter of the final joint model obtained for the TOI-776 system using juliet.

\begin{tabular}{lcc}
\hline \hline Parameter & TOI-776 b & TOI-776 c \\
\hline \multicolumn{3}{c}{ Stellar parameters } \\
$\rho_{\star}\left(\mathrm{kg} \mathrm{m}^{-3}\right)$ & $6024_{-640}^{+650}$ \\
\multicolumn{3}{c}{} \\
$P(\mathrm{~d})$ & Planet parameters \\
$t_{0}^{(a)}$ & $8.24661_{-0.00004}^{+0.00005}$ & $15.6653_{-0.0003}^{+0.0004}$ \\
$r_{1}$ & $8571.4167_{-0.0011}^{+0.0010}$ & $8572.5999_{-0.0016}^{+0.0018}$ \\
$r_{2}$ & $0.43_{-0.07}^{+0.10}$ & $0.51_{-0.07}^{+0.08}$ \\
$e^{(b)}$ & $0.0316_{-0.0011}^{+0.0008}$ & $0.03437_{-0.0008}^{+0.0009}$ \\
$\omega$ & $0.06_{-0.02}^{+0.03}(<0.18)$ & $0.04_{-0.01}^{+0.02}(<0.18)$ \\
$K\left(\mathrm{~m} \mathrm{~s}^{-1}\right)$ & $-67_{-73}^{+117}$ & $-11_{-79}^{+55}$ \\
& $1.88_{-0.44}^{+0.40}$ & $2.05_{-0.68}^{+0.67}$
\end{tabular}

Photometry parameters

$\begin{array}{lc}\sigma_{\text {TESS }}(\mathrm{ppm}) & 1.1_{-1.0}^{+8.0} \\ q_{1, \mathrm{TESS}} & 0.26_{-0.17}^{+0.29} \\ q_{2, \mathrm{TESS}} & 0.36_{-0.20}^{+0.21} \\ \sigma_{\text {LCO-CTIO }}(\mathrm{ppm}) & 1000_{-34}^{+36} \\ M_{\text {LCO-CTIO }}(\mathrm{ppm}) & 890_{-468}^{+395} \\ \theta_{\text {LCO-CTIO }} & 0.0011_{-0.0004}^{+0.0003} \\ q_{1, \mathrm{LCO}-\mathrm{CTIO}} & 0.76_{-0.14}^{+0.13} \\ \sigma_{\text {LCO-SAAO }}(\mathrm{ppm}) & 471_{-60}^{+60} \\ M_{\text {LCO-SAAO }}(\mathrm{ppm}) & -810_{-260}^{+250} \\ \theta_{\text {LCO-SAAO }} & -0.0004 \pm 0.0002 \\ q_{1, \mathrm{LCO}-S A A O} & 0.73_{-0.15}^{+0.14} \\ \sigma_{\text {LCO-SSO }}(\mathrm{ppm}) & 885_{-40}^{+45} \\ M_{\text {LCO-SSO }}(\mathrm{ppm}) & -4057_{-413}^{+386} \\ \theta_{\text {LCO-SSO }} & -0.0031 \pm 0.0003 \\ q_{1, \mathrm{LCO}-\mathrm{SSO}} & 0.46_{-0.18}^{+0.16} \\ \sigma_{\text {MEarth }}(\mathrm{ppm}) & 1734_{-47}^{+52} \\ M_{\text {MEarth }}(\mathrm{ppm}) & -2_{-44}^{+47} \\ q_{1, \mathrm{MEarth}} & 0.73_{-0.14}^{+0.13}\end{array}$

$R V$ parameters

$\begin{array}{ll}\mu_{\text {HARPS }}\left(\mathrm{m} \mathrm{s}^{-1}\right) & 4.33_{-0.58}^{+0.51} \\ \sigma_{\text {HARPS }}\left(\mathrm{m} \mathrm{s}^{-1}\right) & 1.66_{-0.30}^{+0.35}\end{array}$

GP hyperparameters and additional sinusoid

\begin{tabular}{lc}
$\sigma_{\mathrm{GP}, \mathrm{TESS}}(\mathrm{ppm})$ & $0.17_{-0.04}^{+0.06}$ \\
$T_{\mathrm{GP}, \mathrm{TESS}}(\mathrm{d})$ & $0.56_{-0.15}^{+0.19}$ \\
$K\left(\mathrm{~m} \mathrm{~s}^{-1}\right)$ & $2.71_{-0.60}^{+0.53}$ \\
$t_{0}{ }^{(b)}$ & $8607.0_{-12.1}^{+11.6}$ \\
$P(\mathrm{~d})$ & $34.4_{-2.0}^{+1.4}$ \\
\hline
\end{tabular}

Notes. Priors and descriptions for each parameter can be found in Table A.1. ${ }^{(a)}$ Units are BJD $-2450000 .{ }^{(b)} 3 \sigma$ upper limit in parenthesis. 

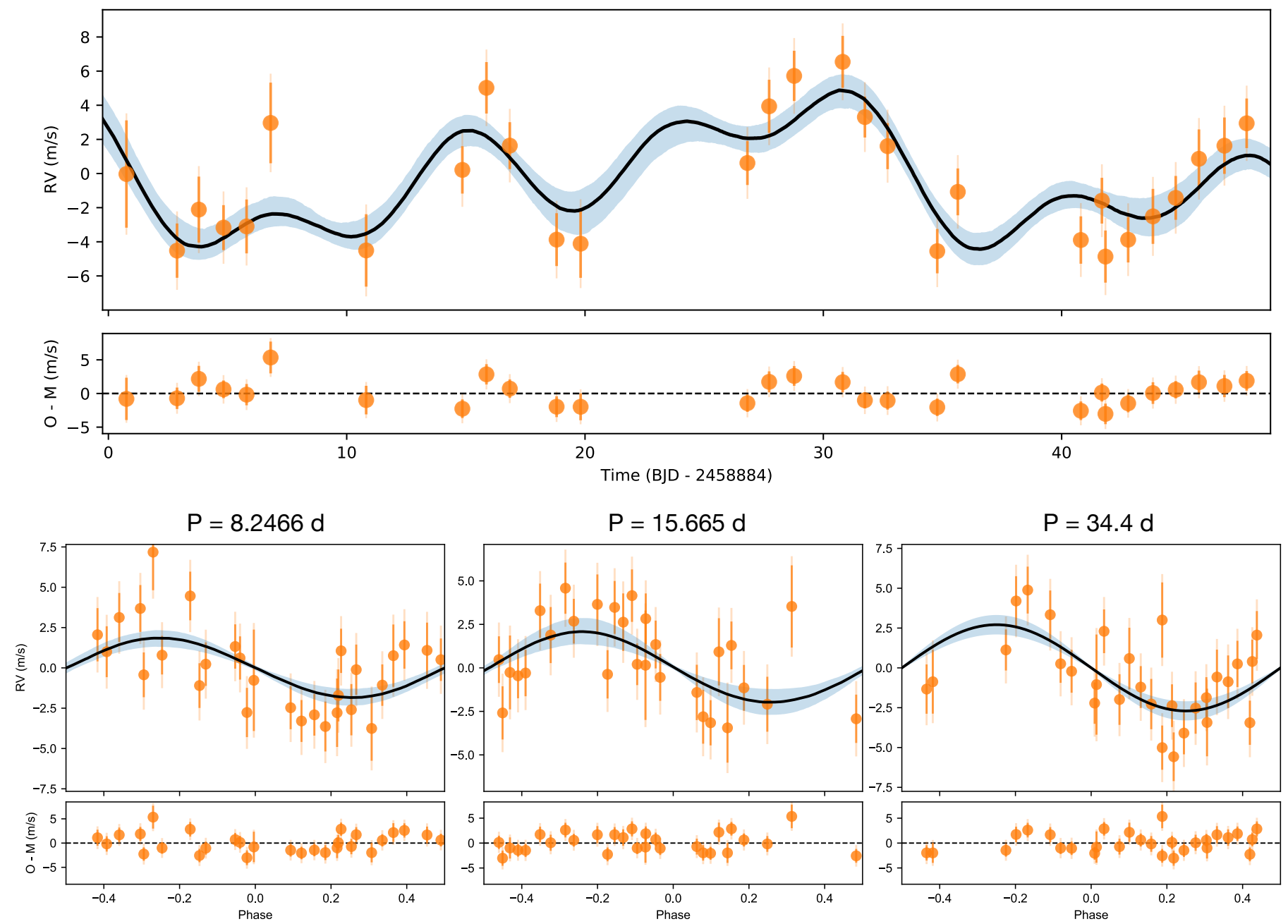

Fig. 8. Top panel: time series of the HARPS serval RVs and the best model discussed in Sect. 5.2.2 and the residuals from the fit below. The blue shaded area corresponds to the $1 \sigma$ confidence interval of the model. Bottom panel: RVs phase-folded to the period (shown above each panel) of the two confirmed planets (TOI-776 b, left; TOI-776 c, center) and the additional sinusoid associated with the stellar variability. In both panels, the error bars of the RV data have the extra jitter term added in quadrature and plotted in a lighter orange for visualization.

bulk density of $3.5_{-1.3}^{+1.4} \mathrm{~g} \mathrm{~cm}^{-3}$. The RV data show only one additional signal, with a semi-amplitude of $\sim 2.7 \mathrm{~m} \mathrm{~s}^{-1}$ and a period of $34 \mathrm{~d}$, associated with the stellar rotation, as suggested by our analyses of the photometry and spectral line indicators.

\subsection{System architecture}

While the occurrence rate of planets around early $\mathrm{M}$ dwarfs $\left(3500 \mathrm{~K}<T_{\text {eff }}<4000 \mathrm{~K}\right)$ has been investigated in detail with Kepler and K2 samples (see e.g., Dressing \& Charbonneau 2013, 2015; Montet et al. 2015; Hirano et al. 2018), the number of currently known planets transiting low-mass stars is still much smaller with respect to those discovered around solar-type stars. While none of these surveys were optimized for M dwarfs, we expect more statistically significant results from the TESS mission for these stars. Figure 9 shows the confirmed transiting planets around $\mathrm{M}$ dwarfs as a function of the orbital period and the effective temperature of the host star. However, very few of these systems have precise determinations of the planetary masses (i.e., densities), eccentricities, and orbital architectures that would be required to link the statistical properties of this population with planet formation and evolution models in the low stellar mass regime.
There are several validated transiting multi-planetary systems orbiting early $\mathbf{M}$ dwarfs similar to TOI-776 in terms of planetary architecture. Kepler-225, Kepler-236, and Kepler231 are two-planet transiting systems composed of super-Earthand/or mini-Neptune-sized companions with similar periods and semi-major axes, all validated by Rowe et al. (2014). However, these systems are on average 5 mag fainter than TOI-776 and the planets do not have a mass determination nor precise stellar parameters. Similarly, K2-240 (Díez Alonso et al. 2018) has two transiting super-Earths with periods of 6 and $20.5 \mathrm{~d}$, although they do not have mass determination, and they orbit an active star that is 2 mag fainter with a clear photometric rotational period of $10.8 \mathrm{~d}$. The two outermost planets of the four-planet system, K2-133, have periods and sizes similar to TOI-776 b and c, but the star is at the faint end for RV follow-up and does not exhibit transit timing variations (TTVs).

If compared to systems with mass determination, TOI-776 shows some similarities with Kepler-26 (Steffen et al. 2012), Kepler-138 (Rowe et al. 2014), TOI-1266 (Demory et al. 2020), and K2-3 (Montet et al. 2015; Crossfield et al. 2015). Kepler-26 b and $\mathrm{c}$ have periods of 12.3 and $17.2 \mathrm{~d}$, respectively, and bulk densities compatible with those of sub-Neptunes determined from TTVs. However, the system has two more planets without mass 

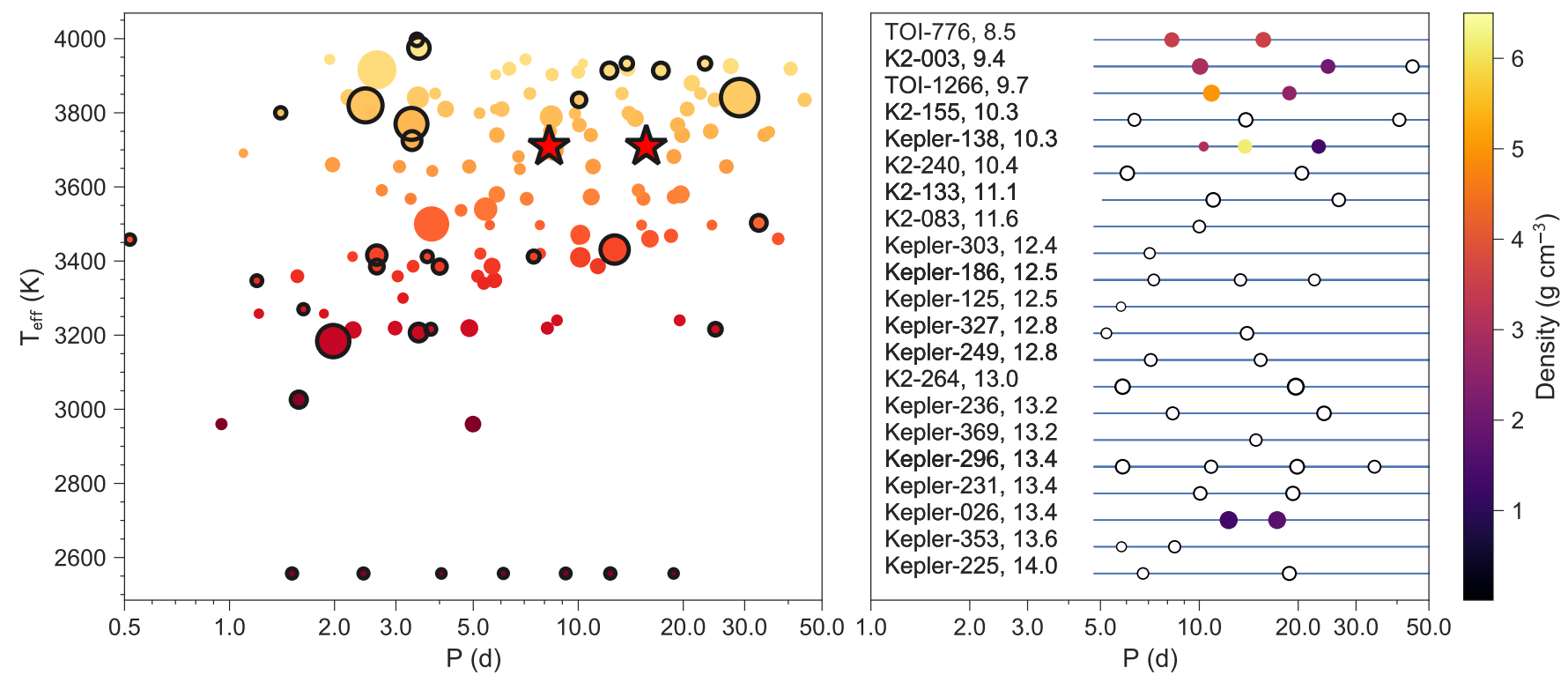

Fig. 9. Left: confirmed transiting planets from the TEPCat database (Southworth 2011) around M dwarfs as a function of period. Black circled points indicate planets with a mass determination better than 30\%. Circles are color-coded by the host effective temperature, and their sizes are proportional to the planet radius. The red stars mark the two planets in the TOI-776 system. Right: transiting multi-planetary systems around earlytype $\mathrm{M}$ dwarfs $\left(3500 \mathrm{~K}<T_{\text {eff }}<4000 \mathrm{~K}\right)$ with similar architectures to the TOI-776 system. Sizes are proportional to the planet radius, and colors indicate their bulk densities. Planets with no mass determination are marked in white. The brightness of the host star in the $J$ band is indicated next to the system's name.

Table 5. Derived planetary parameters obtained for the TOI-776 system using the posterior values from Table 4 and stellar parameters from Table 2 .

\begin{tabular}{lcc}
\hline \hline Parameter $^{(a)}$ & TOI-776 b & TOI-776 c \\
\hline \multicolumn{3}{c}{ Derived transit parameters } \\
$p=R_{\mathrm{p}} / R_{\star}$ & $0.0316_{-0.0011}^{+0.0008}$ & $0.0344_{-0.0008}^{+0.0009}$ \\
$b=\left(a / R_{\star}\right) \cos i_{\mathrm{p}}$ & $0.25_{-0.14}^{+0.10}$ & $0.27_{-0.11}^{+0.12}$ \\
$a / R_{\star}$ & $27.87_{-1.02}^{+0.97}$ & $42.75_{-1.57}^{+1.49}$ \\
$i_{\mathrm{p}}(\mathrm{deg})$ & $89.65_{-0.37}^{+0.22}$ & $89.51_{-0.21}^{+0.25}$ \\
$t_{\mathrm{T}}(\mathrm{h})$ & $2.41_{-0.10}^{+0.11}$ & $2.99_{-0.13}^{+0.16}$
\end{tabular}

Derived physical parameters

\begin{tabular}{lcc}
$M_{\mathrm{p}}\left(M_{\oplus}\right)$ & $4.0 \pm 0.9$ & $5.3 \pm 1.8$ \\
$R_{\mathrm{p}}\left(R_{\oplus}\right)$ & $1.85 \pm 0.13$ & $2.02 \pm 0.14$ \\
$\rho_{\mathrm{p}}\left(\mathrm{g} \mathrm{cm}^{-3}\right)$ & $3.4_{-0.9}^{+1.1}$ & $3.5_{-1.3}^{+1.4}$ \\
$g_{\mathrm{p}}\left(\mathrm{m} \mathrm{s}^{-2}\right)$ & $11.2_{-2.8}^{+3.1}$ & $12.8_{-4.4}^{+4.9}$ \\
$a_{\mathrm{p}}(\mathrm{au})$ & $0.0652 \pm 0.0015$ & $0.1000 \pm 0.0024$ \\
$T_{\mathrm{eq}}(\mathrm{K})^{(b)}$ & $514 \pm 17$ & $415 \pm 14$ \\
$S\left(S_{\oplus}\right)$ & $11.5 \pm 0.6$ & $4.9 \pm 0.2$ \\
\hline
\end{tabular}

Notes. ${ }^{(a)}$ Error bars denote the $68 \%$ posterior credibility intervals. ${ }^{(b)}$ Equilibrium temperatures were calculated assuming zero Bond albedo and uniform surface temperatures across the entire planet.

determination: an inner Earth-sized planet and an outer miniNeptune-sized planet. Kepler-138 is a very interesting system of three small planets, whose densities were estimated through photodynamical modeling (Almenara et al. 2018). The most similar to the TOI-776 planets in terms of orbital period, Kepler-138 b $(10.3 \mathrm{~d})$ and $\mathrm{c}(13.8 \mathrm{~d})$, are very different in composition, the former being a Mars analog and the latter a prototypical rocky super-Earth. The third, outermost planet seems to have retained a substantial volatile-rich envelope. TOI-1266 is the system that resembles TOI-776 the most. The two planets of the system have tentative dynamical masses determined from TTVs, although RVs are likely to become available in the future. The planets straddle the radius valley and, interestingly, the innermost one is larger and more massive than the outer one. K2-3, the brightest of all four systems, has three small transiting planets and only the two inner ones (with periods of 10 and $24.6 \mathrm{~d}$ ) have a mass determination using HARPS-N, HARPS, HIRES and PFS RVs (Almenara et al. 2015; Damasso et al. 2018; Kosiarek et al. 2019), only an upper limit is measured for the third (with a period of $44.5 \mathrm{~d}$ ). The planets have similar compositions, compatible with those of water worlds or water-poor planets with gaseous envelopes; however, the poor bulk density estimations of planets $\mathrm{c}$ and $\mathrm{d}$ impede further conclusions. The right panel of Fig. 9 shows all of the aforementioned systems, color-coded by bulk density and with the $J$-band magnitude of their host stars indicated.

Therefore, we conclude that, although multi-planetary systems of super-Earths and/or sub-Neptunes are common around early-type M dwarfs, only TOI-776 has all of its planets well characterized, bulk density uncertainties better than $30 \%$, precise stellar parameters, and a host star bright enough for atmospheric follow-up observations with current and planned facilities.

\subsection{Dynamics and TTV analysis}

We investigated possible TTVs through a three-body simulation using the Python Tool for Transit Variations (PyTTV; Korth 2020). We simulated the estimated TTVs and RVs using the stellar and planetary parameters reported in Tables 2, 4, and 5 and found an expected TTV signal with a period of $\sim 150 \mathrm{~d}$ and a maximum amplitude of $\sim 2 \mathrm{~min}$ for the inner planet. Thus, the 

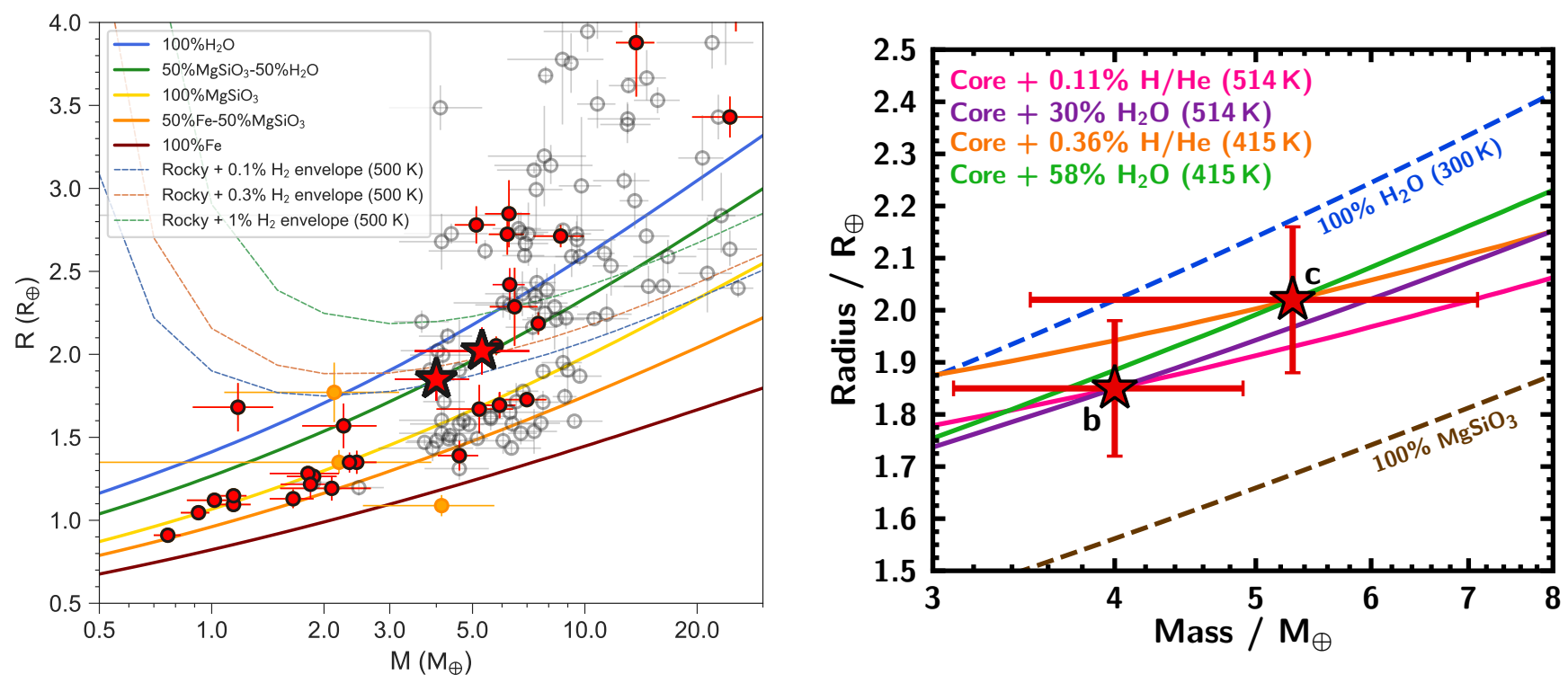

Fig. 10. Mass-radius diagrams in Earth units. In the left panel, open circles are transiting planets around F-, G-, and K-type stars with mass and radius measurements better than 30\% from the TEPCat database of well-characterized planets (Southworth 2011); red circles are planets around $\mathrm{M}$ dwarfs with mass and radius measurements; orange filled circles are planets around $\mathrm{M}$ dwarfs with mass determinations worse than $30 \%$; and the red stars are TOI-776 b and c, which have masses determined with accuracies of 23 and 34\%, respectively. In the left panel, the color lines are the theoretical $R-M$ models of Zeng et al. (2016, 2019). In the right panel, the solid pink and purple lines show the models from Sect. 6.3 that are consistent with the mass and radius of TOI- $776 \mathrm{~b}$, and the orange and green lines show compositions consistent with the mass and radius of TOI-776 c, assuming an Earth-like core (1/3 iron, 2/3 silicates).

time span of the photometric observations, their cadence, and signal-to-noise would prevent the detection of TTVs with the currently available data.

Additionally, we carried out a set of dynamical simulations to study the long-term stability of the system. We used the parameters in Tables 4 and 5 and randomly drew 1000 samples from the posterior distributions as initial parameters for the dynamical simulations. We integrated each parameter set for $10^{6}$ orbits of the inner planet using the REBOUND (Rein \& Liu 2012) tool with the standard IAS15 integrator (Rein \& Spiegel 2015). We also explored the stability using the MEGNO criteria as implemented in REBOUND. In the cases of close encounters between the bodies or a one-body ejection, the system would be flagged as unstable for the specific set of parameters. We found that the system is dynamically stable over the entire integration time and for the whole parameter posterior space.

\subsection{Planetary composition and interior structure}

Figure 10 shows the location of the TOI-776 system in a massradius diagram. Both planets occupy a scarcely populated region, characterized by a lack of planets around $\mathrm{M}$ dwarfs and with precise bulk density measurements. A comparison with the theoretical models by Zeng et al. (2016), reported in the left panel of Fig. 10, shows that TOI-776 b and c are consistent with mixtures of silicates and water in a 50-50 proportion. We adopted the three-layer models from Zeng \& Sasselov (2013) and Zeng et al. (2016) to infer the interior structure of the planets. However, given the mass and radius input, the solution of the model is degenerate. As a consequence, the same mass-radius pair can lead to a broad range of combinations of iron, silicate, and watermass fractions. On the other hand, when we applied the latest models by Zeng et al. (2019), assuming a 1 mbar surface pressure level and an equilibrium temperature of $500 \mathrm{~K}$ (from Table 5), we found that an Earth-like rocky core with a 0.1 and $0.3 \%$ molecular hydrogen atmosphere is consistent with the bulk densities of TOI-776 b and c, respectively. Nonetheless, it is clear that both of the planets in the system have an internal composition ranging from water worlds to rocky planets that have retained a significant atmosphere.

For a better understanding of the nature of the two exoplanets, we performed a more detailed modeling of their interior compositions, using their masses, radii, and surface temperatures. Our model considers a canonical four-layer structure consisting of a two-component iron and silicate core, a layer of $\mathrm{H}_{2} \mathrm{O}$, and a $\mathrm{H} / \mathrm{He}$ envelope. We assume that the core is Earth-like in composition (1/3 iron, $2 / 3$ silicates by mass), meaning the core, water, and $\mathrm{H} / \mathrm{He}$ envelope mass fractions ( $x_{\text {core }}$, $\left.x_{\mathrm{H}_{2} \mathrm{O}}, x_{\mathrm{H} / \mathrm{He}}\right)$ are free parameters that sum to unity. The model solves the planetary structure equations of mass continuity and hydrostatic equilibrium assuming spherical symmetry. Further detail regarding the internal structure model can be found in Madhusudhan et al. (2020) and Nixon \& Madhusudhan (2020).

The equation of state (EOS) prescriptions for the iron and silicate layers are adopted from Seager et al. (2007), who used a Vinet EOS of the $\epsilon$ phase of Fe (Vinet et al. 1989; Anderson et al. 2001) and a Birch-Murnaghan EOS of $\mathrm{MgSiO}_{3}$ perovskite (Birch 1952; Karki et al. 2000). Thermal effects in these layers are ignored, since they have a small effect on the planetary radius (Howe et al. 2014). However, thermal effects in the outer envelope can alter the mass-radius relation significantly (Thomas \& Madhusudhan 2016). For this reason, the model uses a temperature-dependent EOS for the outer $\mathrm{H}_{2} \mathrm{O}$ and $\mathrm{H} / \mathrm{He}$ layers. For $\mathrm{H}_{2} \mathrm{O}$, we used a patchwork EOS in order to cover all possible phases of $\mathrm{H}_{2} \mathrm{O}$ that might be present in the interior, compiled from Salpeter \& Zapolsky (1967); Fei et al. (1993); Wagner \& Pruß (2002); Feistel \& Wagner (2006); Seager et al. (2007); French et al. (2009); Klotz et al. (2017), and Journaux et al. (2020). For H/He, we used the EOS in Chabrier et al. (2019), which assumes a solar helium fraction $(Y=0.275)$. The 
temperature profile in the envelope is isothermal from the surface down to some radiative-convective boundary, where it becomes adiabatic. The pressure at the radiative-convective boundary $P_{\mathrm{rc}}$ is a free parameter in the model. For this study, we considered values of $P_{\text {rc }}$ ranging from 1-100 bar.

We explored the parameter space of possible compositions in $\left(x_{\text {core }}, x_{\mathrm{H}_{2} \mathrm{O}}, x_{\mathrm{H} / \mathrm{He}}\right)$ space. For each composition, we considered a range of masses that agree with the observed mass of the planet to within $1 \sigma$. For a given mass $\hat{M}$, the model radius $\hat{R}$ is computed, and the $\chi^{2}$ statistic is calculated:

$\chi^{2}=\frac{\left(M_{\mathrm{p}}-\hat{M}\right)^{2}}{\sigma_{M}^{2}}+\frac{\left(R_{\mathrm{p}}-\hat{R}\right)^{2}}{\sigma_{R}^{2}}$,

where $\left(\sigma_{M}, \sigma_{R}\right)$ are the observed uncertainties on the mass and radius of each planet.

The bulk densities of TOI-776 $\mathrm{b}$ and $\mathrm{c}\left(3.4_{-0.9}^{+1.1} \mathrm{~g} \mathrm{~cm}^{-3}\right.$ and $3.5_{-1.3}^{+1.5} \mathrm{~g} \mathrm{~cm}^{-3}$, respectively) are too low for either planet to have a purely terrestrial (iron plus rock) composition. Therefore, the planets must possess an envelope with some amount of $\mathrm{H}_{2} \mathrm{O}$ and/or $\mathrm{H} / \mathrm{He}$, in order to explain their masses and radii. The right panel of Fig. 10 shows limiting cases for each planet in which the envelope composition is either purely $\mathrm{H}_{2} \mathrm{O}$ or purely $\mathrm{H} / \mathrm{He}$. The mass and radius of TOI-776 $\mathrm{b}$ can be explained to within $1 \sigma$ $\left(\chi^{2} \leq 1\right)$ with a pure $\mathrm{H}_{2} \mathrm{O}$ envelope of $12-73 \%$ by mass or a pure $\mathrm{H} / \mathrm{He}$ envelope with a mass fraction of $1.1 \times 10^{-4}-5.2 \times 10^{-3}$. Best-fit solutions (those which minimize $\chi^{2}$ ) for pure envelopes are found at $x_{\mathrm{H}_{2} \mathrm{O}}=0.3$ and $x_{\mathrm{H} / \mathrm{He}}=1.1 \times 10^{-3}$. TOI-776 c might have larger envelopes; within $1 \sigma$, it is consistent with a pure $\mathrm{H}_{2} \mathrm{O}$ layer of $\geq 18 \%$ or a pure $\mathrm{H} / \mathrm{He}$ envelope with a mass fraction of $5.4 \times 10^{-4}-1.2 \times 10^{-2}$. The best-fit pure-envelope solutions for TOI-776 $\mathrm{c}$ are $x_{\mathrm{H}_{2} \mathrm{O}}=0.58$ and $x_{\mathrm{H} / \mathrm{He}}=3.6 \times 10^{-3}$. Each of the best-fit models, shown in the right panel of Fig. 10, have a radiative-convective boundary at $P_{\mathrm{rc}}=10 \mathrm{bar}$.

It is also possible that the planets in this system have both $\mathrm{H}_{2} \mathrm{O}$ and $\mathrm{H} / \mathrm{He}$ components, as well as an iron/rock core. For the three components, we explored the full range of plausible values $\left(x_{\text {core }}, x_{\mathrm{H}_{2} \mathrm{O}}\right.$, and $x_{\mathrm{H} / \mathrm{He}}$ ) that could explain the interior compositions of each planet. We considered two different temperature profiles for each planet, with $P_{\mathrm{rc}}=1$ and 100 bar. Figure 11 shows the mass fractions of water and $\mathrm{H} / \mathrm{He}$ compatible to within $1 \sigma$ $\left(\chi^{2} \leq 1\right)$ with the masses and radii of TOI-776 b and c. We obtained upper limits on the total $\mathrm{H}_{2} \mathrm{O}$ and $\mathrm{H} / \mathrm{He}$ mass fractions for TOI-776 b: $x_{\mathrm{H}_{2} \mathrm{O}} \leq 73 \%$ and $x_{\mathrm{H} / \mathrm{He}} \leq 0.52 \%$. These correspond to cases with pure $\mathrm{H}_{2} \mathrm{O}$ or $\mathrm{H} / \mathrm{He}$ envelopes, as previously discussed. For TOI-776 c, we find that $x_{\mathrm{H} / \mathrm{He}} \leq 1.2 \%$. A $100 \%$ $\mathrm{H}_{2} \mathrm{O}$ planet would theoretically be consistent with the mass and radius of TOI-776 c, but this would be unrealistic from a planet formation perspective, as some rocky material is needed for further accretion of ice and gas (Lee \& Chiang 2016). Figure 11 also shows a significant overlap between the best-fit shaded regions for the two planets, meaning that the planets could also share the same composition.

The masses and radii of TOI-776 $\mathrm{b}$ and $\mathrm{c}$ allow for a wide range of possible solutions, from water worlds with steam atmospheres to mostly rocky planets with hydrogen-rich envelopes; however, they are inconsistent with bare rocks without atmospheres. Our models assume a surface pressure of $0.1 \mathrm{bar}$, meaning a water-world solution for either planet yields a steam atmosphere. On the other hand, a higher surface pressure could result in liquid $\mathrm{H}_{2} \mathrm{O}$ at the surface. A rocky planet with an outgassed secondary atmosphere which includes carbon compounds is unlikely: Elkins-Tanton \& Seager (2008) placed an upper limit on the mass fraction for this type of atmosphere at $5 \%$. The lower

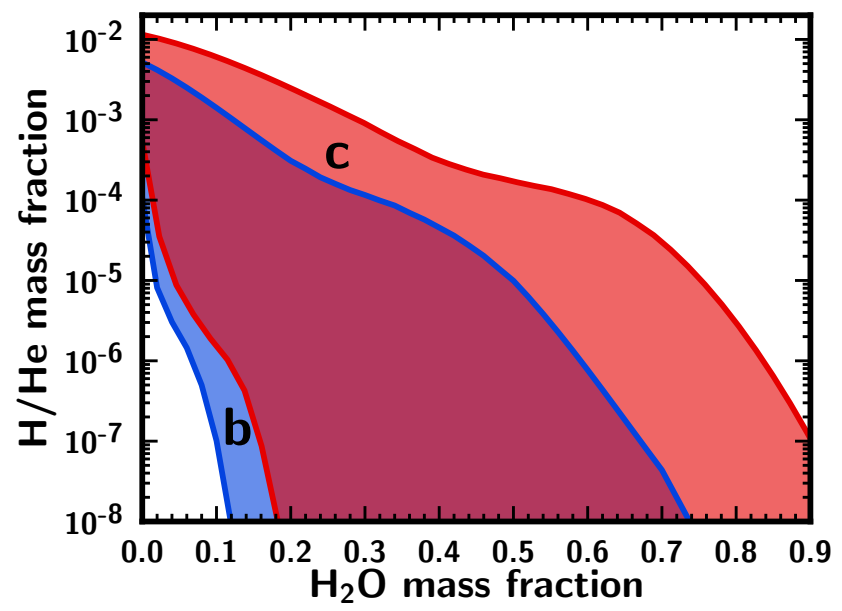

Fig. 11. $\mathrm{H} / \mathrm{He}$ vs. $\mathrm{H}_{2} \mathrm{O}$ mass fractions for the best-fit interior compositions $(\leq 1 \sigma)$ permitted by the masses and radii of TOI-776 $\mathrm{b}$ and c, assuming an Earth-like core, for two different pressure-temperature profiles with radiative-convective boundaries at 1 and 100 bar. The blue shaded region indicates possible compositions for TOI-776 b, and the red shaded region shows compositions for TOI-776 c. The darker red shaded area between the two corresponds to the range of possible compositions that could explain both planets. For TOI-776 b, the $\mathrm{H}_{2} \mathrm{O}$ mass fraction is constrained to be $\leq 73 \%$ and the $\mathrm{H} / \mathrm{He}$ mass fraction is $\leq 0.52 \%$. For TOI-776 c the upper limit for $\mathrm{H} / \mathrm{He}$ is $1.2 \%$. A purely $\mathrm{H}_{2} \mathrm{O}$ planet would be consistent with this mass and radius, but we only show $\mathrm{H}_{2} \mathrm{O}$ mass fractions up to $90 \%$.

mass limits in the case of pure $\mathrm{H}_{2} \mathrm{O}$ envelopes are 8 and $18 \%$ for TOI-776 b and c, respectively. On the other hand, in a carbonrich atmosphere, the dominant species, $\mathrm{CO}_{2}$, has a higher mean molecular weight than $\mathrm{H}_{2} \mathrm{O}$, leading to a lower atmospheric scale height. All things considered, we can infer that a 5\% carbonrich atmosphere is less than what would be needed to explain the planet radii. However, determining whether the two planets have $\mathrm{H}_{2} \mathrm{O}$ - or $\mathrm{H} / \mathrm{He}$-rich atmospheres is impossible with the present data. Atmospheric observations of the planets would be required in order to break this degeneracy.

\subsection{Radius gap in M dwarfs}

The occurrence rate distribution of close-in planets exhibits a paucity of planets from 1.7-2.0 $R_{\oplus}$ (Fulton et al. 2017; Fulton \& Petigura 2018; Hardegree-Ullman et al. 2020) around FGK stars $\left(T_{\text {eff }}>4700 \mathrm{~K}\right.$ ), and from 1.4-1.7 $R_{\oplus}$ (Hirano et al. 2018; Cloutier \& Menou 2020) around mid-K to mid-M dwarfs $\left(T_{\text {eff }}<\right.$ $4700 \mathrm{~K})$. This feature is pointed out as the result of the transition from small rocky planets to larger non-rocky planets with envelopes rich in volatiles (Weiss \& Marcy 2014; Dressing \& Charbonneau 2015). Recent studies showed that the location of the radius gap depends on the orbital period, or, alternatively, on the planet's insolation (Van Eylen et al. 2018; Martinez et al. 2019; Cloutier \& Menou 2020). Additionally, the width and center of the radius gap also depends on whether the host star is single or part of a multiple star system (Teske et al. 2018).

According to the above discussion, if we consider the radius axis in Fig. 10, TOI-776 b and c belong, within the uncertainties, to the radius gap in the case of FGK stars. On the other hand, they are well above the radius gap if we account for mid-K to mid-M dwarfs. Similarly, when looking at the distribution of transiting planets in a radius-insulation diagram (Fig. 12, left panel), the TOI-776 planets lie above the radius valley - the $2 \mathrm{D}$ view of 

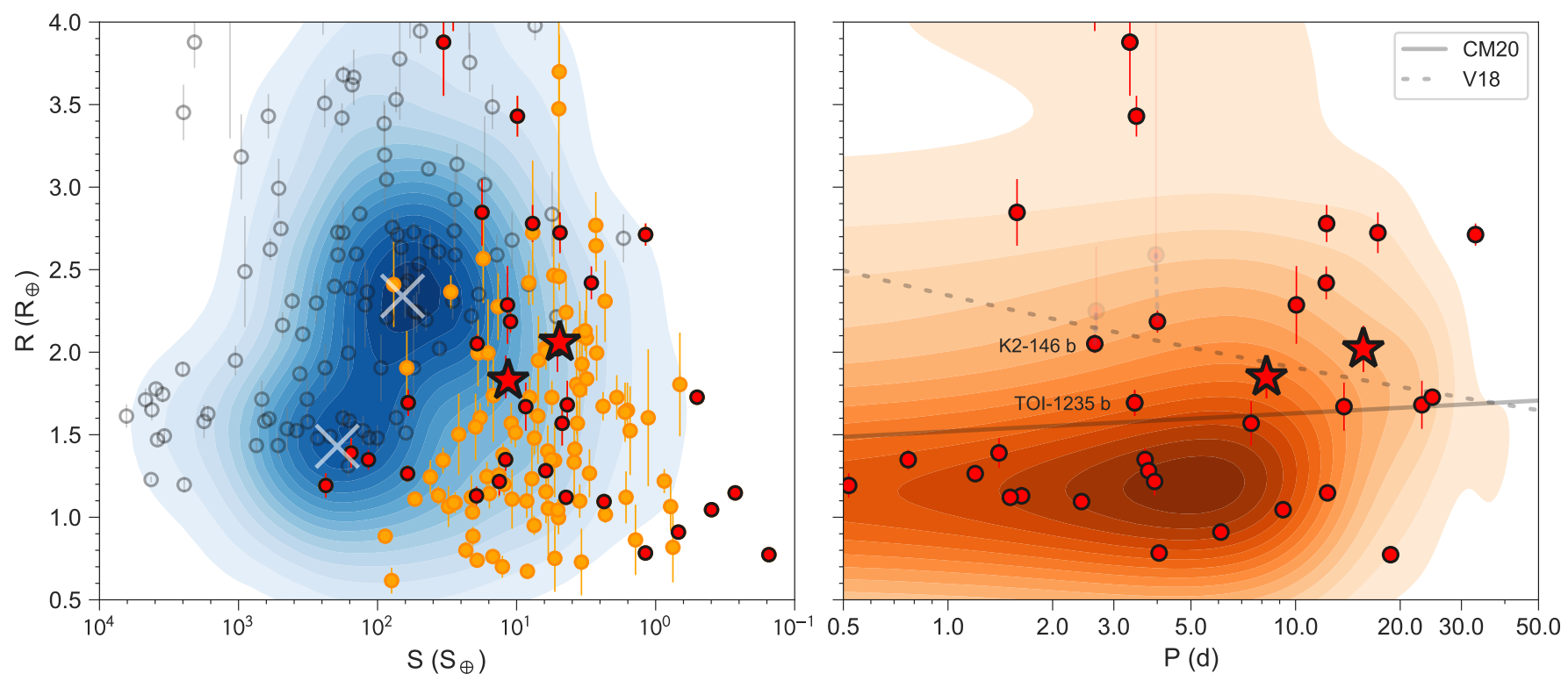

Fig. 12. Insolation-radius (left) and period-radius (right) diagrams in Earth units. In both panels, the different circles represent the same planets as in Fig. 10 from the TEPCat database (Southworth 2011). In the left panel, we plot in blue the $R-S$ point density of all the known confirmed transiting planets with contours, and sub-Neptune and super-Earths' density maxima with white crosses. In the right panel, the orange contours represent the density of planets around $\mathrm{M}$ dwarfs with mass determinations worse than $30 \%$ or without mass constraints at all (orange circles in the left panel). The dashed line represents the location of the radius valley for F-, G-, and K-type stars from Van Eylen et al. (2018), consistent with the predictions from photoevaporation and core-powered mass-loss models, while the solid line represents the location of the radius valley for mid-K and mid-M dwarfs from Cloutier \& Menou (2020), consistent with gas-poor formation scenarios. Together with TOI-776 b, the other two systems that are within both lines are K2-146 (solid, Hamann et al. 2019; Lam et al. 2020, translucent) and TOI-1235 (Bluhm et al. 2020; Cloutier et al. 2020).

the radius gap - that separates rocky super-Earths from gaseous sub-Neptunes around FGK stars.

The right panel of Fig. 12 shows the period-radius diagram of all known exoplanets with precise bulk density measurements that orbit M dwarfs. The dashed line marks the empirical location of the radius valley for FGK stars, following Van Eylen et al. (2018), while the solid line indicates the location of mid-K to mid-M dwarfs as in Cloutier \& Menou (2020). The change in slope as a function of stellar type is the result of a change in the dominant mechanism responsible for sculpting the radius valley. For instance, the thermally driven mass loss, caused by photoevaporation or core-powered mechanisms, becomes less efficient toward low-mass stars. The measured slope for mid-K to mid-M dwarfs suggests that gas-poor formation (Lee et al. 2014; Lee \& Chiang 2016; Lopez \& Rice 2018) might be the main process by which small planets form. However, thousands of small planets around low-mass stars with precise radii are needed in order to robustly state if the radius valley is the result of the erosion or the gas-poor formation scenarios (Cloutier et al. 2020). Although enriching the sample of exoplanet systems orbiting $M$ dwarfs is nowadays possible thanks to TESS and future space-based missions such as PLATO, an alternative is to obtain precise bulk density measurements of exoplanets lying in the region of discrepancy between models.

Planet TOI-776 b joins TOI-1235 b (Bluhm et al. 2020; Cloutier et al. 2020) and K2-146 b (Lam et al. 2020; Hamann et al. 2019) inside the period-radius region, where thermally driven mass-loss models disagree with the predictions from gaspoor formation. However, K2-146 b belongs to this region if we refer to the parameters reported in Hamann et al. (2019), because the radius estimated by Lam et al. (2020) (see translucent points in Fig. 12) is more than $2 \sigma$ higher, causing the planet to be placed outside the radius valley. Our previous analyses show that both TOI-776 b and c are likely to have retained a significant atmosphere, with slightly different envelope mass fractions. This result, given their periods and radii, would be consistent with the predictions from gas-poor formation models.

On the other hand, the system's composition may be reconciled with thermally driven mass loss because the inner, most irradiated planet has a smaller envelope mass fraction compared to its outer companion. Unlike other known systems whose planets straddle both sides of the radius gap (e.g., Dumusque et al. 2014; Niraula et al. 2017; Nowak et al. 2020), TOI-776 is an interesting case where photo-evaporation could have stopped or become inefficient early in the planet's history. However, it is possible that the planets are currently undergoing mass loss under the core-powered mechanism, which erodes sub-Neptune planets into rocky super-Earths on Gyr timescales (Ginzburg et al. 2018), contrary to the few Myr timescale during which photoevaporation is effective (Sanz-Forcada et al. 2011). As reported in Table 2, the age of TOI-776 is between 2 and 10 Gyr. However, the current data precision and limited number of known planets in this specific regime hamper any further investigation in favor of one or the other mechanism of formation. New studies on the dependence of the radius valley with other stellar parameters such as the age or metallicity, together with a larger sample of well-characterized planets in or near the radius valley, will help to discern between them in a demographic sense (HardegreeUllman et al. 2020; Berger et al. 2020; Gupta \& Schlichting 2020).

However, for the first time, we can compare planets that belong to this region of the parameter space where formation models make opposing predictions. TOI-1235 b has a rocky composition with a $90 \%$ confidence upper limit in the envelope mass fraction of $0.5 \%$, thus incompatible with a gas-poor formation scenario. We reach the opposite conclusion for TOI-776 b 

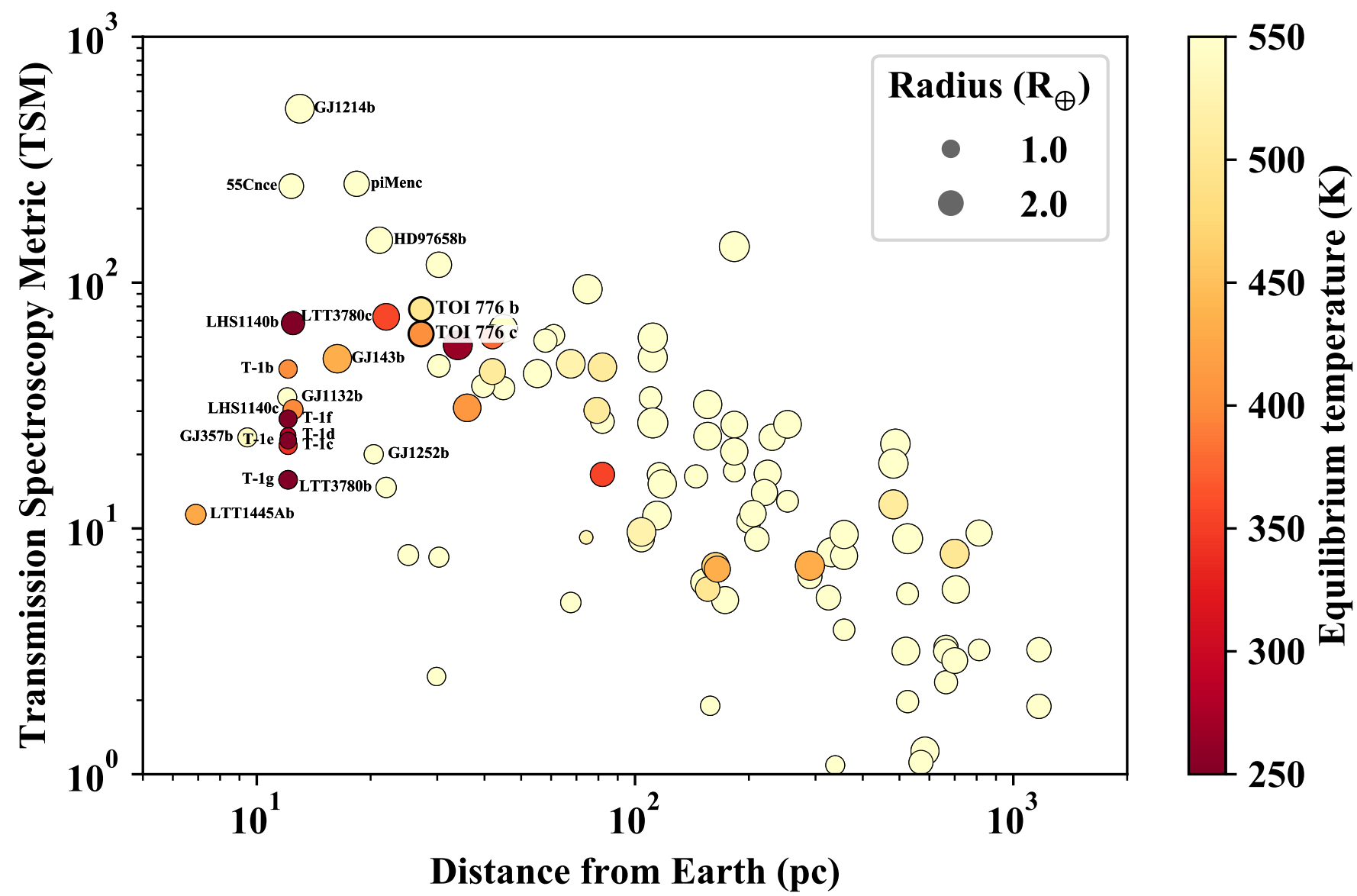

Fig. 13. Transmission spectroscopy metric (TSM) for exoplanets from the Exoplanet Encyclopedia with a radius below $3 R_{\oplus}$ and a mass determination by RVs or TTVs. TOI-776 b and $\mathrm{c}$ are labeled and marked with thicker black borderlines.

and $\mathrm{c}$, whose bulk densities imply the presence of a volatile envelope making them compatible with the predictions from gas-poor formation mechanisms, given their periods and radii. Therefore, although other stellar parameters might need to be taken into account, we can tentatively predict that the stellar mass below which thermally driven mass loss is no longer the main formation pathway for sculpting the radius valley is probably between 0.63 and $0.54 M_{\odot}$, which corresponds to the host stellar masses of TOI-1235 and TOI-776, respectively. More planets in this interesting region of the parameter space with precise bulk density measurements are key to revealing the mechanisms responsible for the radius valley emergence around low-mass stars with respect to solar-like stars.

\subsection{Atmospheric characterization}

\subsubsection{Transmission spectroscopy metric}

We used the proposed metric by Kempton et al. (2018) to evaluate the suitability of the TOI-776 planets for atmospheric characterization studies. Figure 13 shows the transmission spectroscopy metric (TSM) for all exoplanets in the Exoplanet Encyclopedia ${ }^{7}$ with a radius below $3 R_{\oplus}$. We used the scale factors listed in Table 1 from Kempton et al. (2018) as opposed to the suggested value for temperate planets, 0.167 , to compute the TSM values in Fig. 13. The estimated TSM of TOI-776 b and $c$ are 77.9 and 61.8, respectively, which places them among the top priority targets

\footnotetext{
7 wwW.exoplanet.eu
}

for atmospheric follow-ups of small planets around nearby stars. This is not surprising, because TOI-776 is one of the brightest $\mathrm{M}$ dwarfs with known transiting planets. However, most of the planets shown in Fig. 13 are well below the radius gap, which makes the TOI-776 system a valuable target for atmospheric characterization in order to trace the formation and evolution of multi-planetary systems orbiting low-mass stars and break the degeneracy of internal composition models.

\subsubsection{Synthetic spectra}

In order to quantitatively assess the possibility of TOI-776 b and c's atmospheric characterization with the James Webb Space Telescope (JWST), we investigated a suite of atmospheric scenarios and calculated their JWST synthetic spectra using the photochemical model ChemKM (Molaverdikhani et al. 2019a) and petitRADTRANS (Mollière et al. 2019). We based the temperature structure of these planets on modern Earth's temperature structure, and we increased the surface temperature for it to be consistent with the equilibrium temperature of TOI776 b and c (Kawashima \& Rugheimer 2019). We followed a similar approach as in Luque et al. (2019): we estimated TOI-776's ( $\left.T_{\text {eff }}=3709 \mathrm{~K}\right)$ flux in the range between X-rays and optical wavelengths, using GJ 832's geometric mean spectra $\left(T_{\text {eff }}=3816 \mathrm{~K}\right)$ as a reference. The stellar data were obtained from the MUSCLES database (France et al. 2016). To set up the models, we used the chemical network of Hébrard et al. (2012) with 135 species and 788 reactions, and UV absorption 


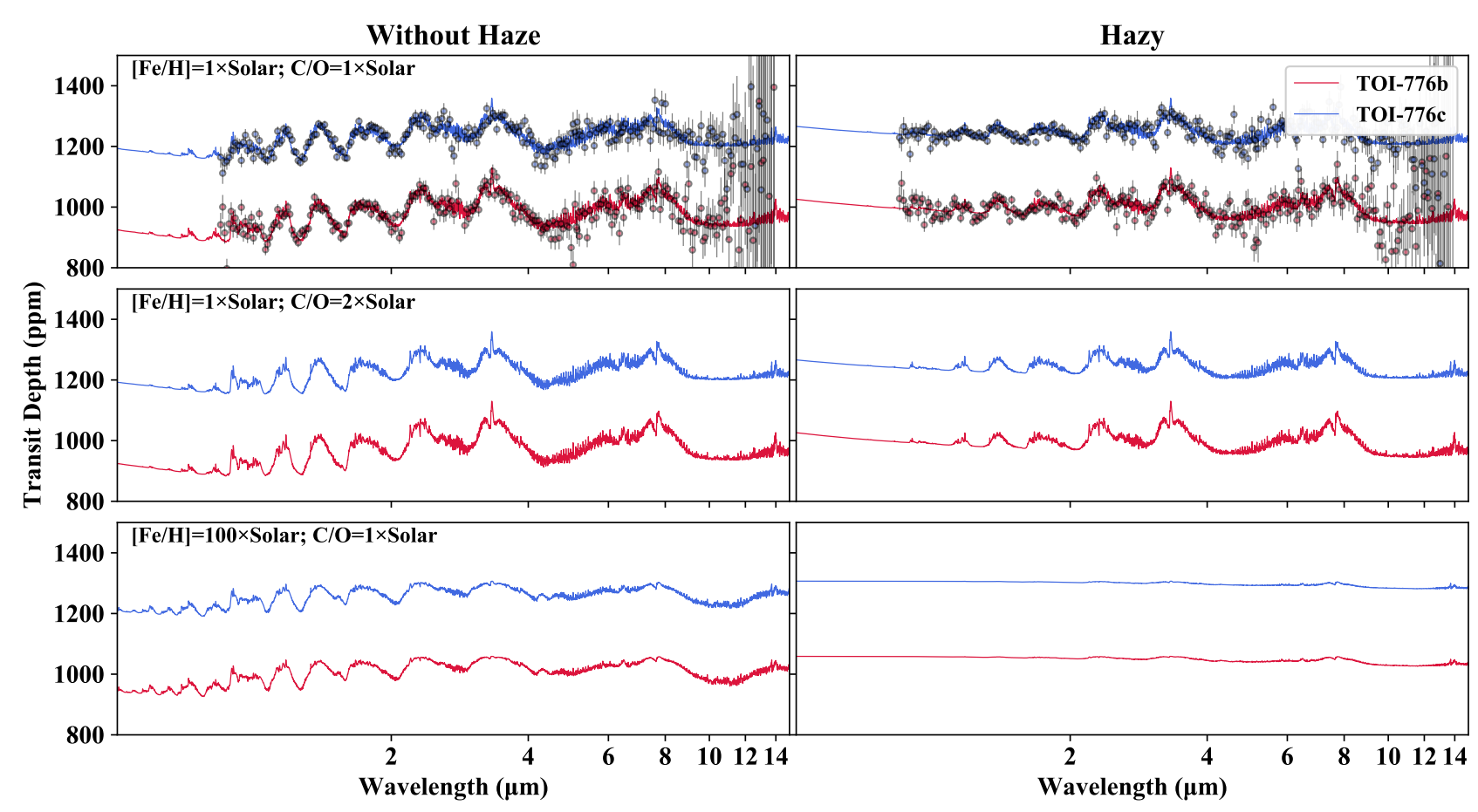

Fig. 14. Synthetic atmospheric spectra of TOI-776 b (red) and c (blue). Top: fiducial models with solar abundance (solid lines). Estimated uncertainties are shown for JWST NIRISS-SOSS, NIRSpec-G395M, and MIRI-LRS configurations, assuming two transits and binned for $R=50$. Middle: enhanced carbon-to-oxygen ratio by a factor of two. Bottom: enhanced metallicity by a factor of 100 . The left column represents spectra without haze opacity, and the right column with haze opacity.

cross-sections and branching yields from an updated version of Hébrard et al. (2012).

Figure 14 shows the synthetic transmission spectra of TOI-776 b and c assuming different metallicities, carbon-tooxygen ratios, and haze opacities. For our fiducial model (top-left panel of Fig. 14), we assume solar abundances. Such spectra predominantly consist of water and methane features, as expected for this type of planet (Molaverdikhani et al. 2019b). The significance of these features are of the order of $100 \mathrm{ppm}$, well above the conservative JWST expected noise floor (20 ppm for NIRISS and $50 \mathrm{ppm}$ for MIRI, Greene et al. 2016). We calculated the NIRISS-SOSS, NIRSpec-G395M, and MIRI-LRS uncertainties with PandExo (Batalha et al. 2017), assuming two transits and binned for $R=50$, supporting the previous statement. In this scenario, the contribution from haze opacity partially obscures molecular features below $2 \mu \mathrm{m}$, but it is almost ineffective at longer wavelengths (see left and right upper panels of Fig. 14). We note, however, that the radiative feedback of haze particles might significantly affect the temperature structure and the composition of atmosphere (Molaverdikhani et al. 2020). We did not take this effect into account in this work in order to keep the temperature profiles consistent with the Earth's profile.

Smaller planets are expected to have enhanced metallicities (e.g. Wakeford et al. 2017). Therefore, we investigated two deviations from our solar abundance fiducial model: (1) an enhanced carbon-to-oxygen ratio $(\mathrm{C} / \mathrm{O})$ two-times the solar value; and (2) an enhanced metallicity one-hundred-times higher than solar. $\mathrm{C} / \mathrm{O}$ enhancement alone does not affect the composition and spectral features substantially, as seen in the middle panels of Fig. 14. On the other hand, one might expect a higher metallicity to result in more pronounced spectral features, due to higher species abundances. However, the bottom panels of Fig. 14 discard this possibility. On the contrary, an enhanced metallicity causes a higher mean molecular weight, which in turn shrinks the spectral significance (bottom-left panel of Fig. 14), and, simultaneously, it results in a higher haze production, which also obscures the spectra significantly (bottom-right panel of Fig. 14). Therefore, a flat transmission spectrum may indicate a hazy atmosphere with a high metallicity (Kreidberg et al. 2014) as opposed to a nonexistent atmosphere (Kreidberg et al. 2019). Complementary observations, such as ground-based highresolution spectroscopy or spectroscopy of the reflected light, are required to reveal the true nature of these flat spectra.

\section{Summary}

We present the discovery and characterization of the two-planet system transiting the bright $(V=11.54 \mathrm{mag}, J=8.48 \mathrm{mag}) \mathrm{M} 1 \mathrm{~V}$ star TOI-776. Both planets were detected by the TESS mission, confirmed from ground-based transit follow-up observations and have had their dynamical masses determined with precise RV measurements using HARPS. In addition, fifteen years of ground-based photometric monitoring by ASAS-SN, ASAS, NSVS, Catalina, and SuperWASP helped us to measure a rotational period between 30 and $40 \mathrm{~d}$, typical of inactive early-type M dwarfs. Our findings are summarized below:

- A joint fit of all the available transit photometry from TESS, MEarth, and LCOGT, and the precise RVs from HARPS reveal that the TOI-776 system consists of two transiting planets, namely TOI-776 b, which has a period of $8.25 \mathrm{~d}$, a radius of $1.85 \pm 0.13 R_{\oplus}$, a mass of $4.0 \pm 0.9 M_{\oplus}$, bulk density of $3.4_{-0.9}^{+1.1} \mathrm{~g} \mathrm{~cm}^{-3}$, and an equilibrium temperature of $514 \pm 17 \mathrm{~K}$; and TOI-776 c, which has a period of $15.66 \mathrm{~d}$, a radius of $2.02 \pm 0.14 R_{\oplus}$, a mass of $5.3 \pm 1.8 M_{\oplus}$, a bulk density of $3.5_{-1.3}^{+1.4} \mathrm{~g} \mathrm{~cm}^{-3}$, and an equilibrium temperature of $415 \pm 14 \mathrm{~K}$. The RV data show one additional signal, with a period of $34 \mathrm{~d}$, associated with the star's rotation, in 
agreement with our analyses of the photometry and spectral line indicators.

- The bulk densities of TOI-776 b and c allow for a wide range of possible interior compositions, from water worlds to rocky planets with $\mathrm{H} / \mathrm{He}$-rich atmospheres, but they are too low for either planet to have a purely terrestrial (iron plus rock) composition. Thus, an atmosphere is expected for both planets.

- From its location in a period-radius diagram, TOI-776 b lies in the transition region where formation and evolution models make different predictions for planetary systems orbiting M dwarfs. For the TOI-776 system, the planets lie above the radius valley carved by gas-poor formation mechanisms, in agreement with their bulk densities being incompatible with the absence of an atmosphere. Still, it is possible that the planets are still undergoing slow thermally driven mass loss under the core-powered scenario.

The TOI-776 system is an excellent target for the JWST. It is the only known multi-planetary system with planets inside and near the radius valley for which all planets: (1) have a bulk density determination with at least $30 \%$ relative uncertainties; and (2) are perfect candidates for atmospheric characterization. Thanks to the brightness of its host star, it is a remarkable laboratory in which to break the degeneracy in planetary interior models and to test formation and evolution theories of small planets around low-mass stars.

Acknowledgements. This work was supported by the KESPRINT ${ }^{8}$ collaboration, an international consortium devoted to the characterization and research of exoplanets discovered with space-based missions. We are very grateful to the ESO staff members for their precious support during the observations. We warmly thank Xavier Dumusque and François Bouchy for coordinating the shared observations with HARPS and Jaime Alvarado Montes, Xavier Delfosse, Guillaume Gaisné, Melissa Hobson, and Felipe Murgas who helped collecting the data. This paper includes data collected by the TESS mission. Funding for the TESS mission is provided by the NASA Explorer Program. We acknowledge the use of TESS Alert data, which is currently in a beta test phase, from pipelines at the TESS Science Office and at the TESS Science Processing Operations Center. Resources supporting this work were provided by the NASA High-End Computing (HEC) Program through the NASA Advanced Supercomputing (NAS) Division at Ames Research Center for the production of the SPOC data products. This research has made use of the Exoplanet Follow-up Observation Program website, which is operated by the California Institute of Technology, under contract with the National Aeronautics and Space Administration under the Exoplanet Exploration Program. This work has made use of data from the European Space Agency (ESA) mission Gaia (https://www. cosmos.esa.int/gaia), processed by the Gaia Data Processing and Analysis Consortium (DPAC, https://www. cosmos.esa.int/web/gaia/dpac/ consortium). Funding for the DPAC has been provided by national institutions, in particular the institutions participating in the Gaia Multilateral Agreement The MEarth Team gratefully acknowledges funding from the David and Lucile Packard Fellowship for Science and Engineering (awarded to D.C.). This material is based upon work supported by the National Science Foundation under grants AST-0807690, AST-1109468, AST-1004488 (Alan T. Waterman Award), and AST-1616624, and upon work supported by the National Aeronautics and Space Administration under Grant No. 80NSSC18K0476 issued through the XRP Program. This work is made possible by a grant from the John Templeton Foundation. The opinions expressed in this publication are those of the authors and do not necessarily reflect the views of the John Templeton Foundation. This work makes use of observations from the LCOGT network. Some of the Observations in the paper made use of the High-Resolution Imaging instrument Zorro. Zorro was funded by the NASA Exoplanet Exploration Program and built at the NASA Ames Research Center by Steve B. Howell, Nic Scott, Elliott P. Horch, and Emmett Quigley. Data were reduced using a software pipeline originally written by Elliott Horch and Mark Everett. Zorro was mounted on the Gemini South telescope, and NIRI was mounted on the Gemini North telescope, of the international Gemini Observatory, a program of NSF's OIR Lab, which is managed by the Association of Universities for Research in Astronomy (AURA) under a cooperative agreement with the National Science Foundation on behalf of the Gemini partnership: the National Science Foundation (United States), National

8 wWW.kesprint.science
Research Council (Canada), Agencia Nacional de Investigación y Desarrollo (Chile), Ministerio de Ciencia, Tecnología e Innovación (Argentina), Ministério da Ciência, Tecnologia, Inovações e Comunicações (Brazil), and Korea Astronomy and Space Science Institute (Republic of Korea). Data collected under program GN-2019A-LP-101. Based in part on observations obtained at the Southern Astrophysical Research (SOAR) telescope, which is a joint project of the Ministério da Ciência, Tecnologia e Inovações (MCTI/LNA) do Brasil, the US National Science Foundation's NOIRLab, the University of North Carolina at Chapel Hill (UNC), and Michigan State University (MSU). This work was enabled by observations made from the Gemini North telescope, located within the Maunakea Science Reserve and adjacent to the summit of Maunakea. We are grateful for the privilege of observing the Universe from a place that is unique in both its astronomical quality and its cultural significance. Based on observations collected at the European Organization for Astronomical Research in the Southern Hemisphere under ESO programme 0103.C-0449(A). R.L. has received funding from the European Union's Horizon 2020 research and innovation program under the Marie Skłodowska-Curie grant agreement No. 713673 and financial support through the "la Caixa" INPhINIT Fellowship Grant LCF/BQ/IN17/11620033 for Doctoral studies at Spanish Research Centers of Excellence from "la Caixa" Banking Foundation, Barcelona, Spain. This work is partly financed by the Spanish Ministry of Economics and Competitiveness through projects ESP2016-80435-C2-2-R and ESP2016-80435-C2-1-R L.M.S. and D.G. gratefully acknowledge financial support from the CRT foundation under Grant No. 2018.2323 "Gaseous or rocky? Unveiling the nature of small worlds". This work is supported by JSPS KAKENHI Grant Numbers JP19K14783, JP18H01265 and JP18H05439, and JST PRESTO Grant Number JPMJPR1775. I.J.M.C. acknowledges support from the NSF through grant AST1824644. J.K., Sz.Cs., M.E., A.P.H., K.W.F.L., S.G. acknowledge support by DFG grants PA525/ 18-1, PA525/ 19-1, PA525/ 20-1, HA3279/ 12-1 and RA714/ 14-1 within the DFG Schwerpunkt SPP 1992, Exploring the Diversity of Extrasolar Planets. M.F, I.G. and C.M.P. gratefully acknowledge the support of the Swedish National Space Agency (DNR 65/19 and 174/18). P.K. and J.S. acknowledge the grant INTER-TRANSFER number LTT20015. S.A. knowledges support from the Danish Council for Independent Research, through a DFF Sapere Aude Starting Grant no. 4181-00487B. Funding for the Stellar Astrophysics Centre is provided by The Danish National Research Foundation (Grant agreement no.: DNRF106). H.J.D. acknowledges support by grant ESP2017-87676-C5-4-R of the Spanish Secretary of State for R\&D\&i (MINECO). D.G. warmly thanks Javier Alarcón, Pablo Arias, Duncan Castex, Mónica Castillo, Cecilia Farias, Mario Herrera, Francisco Labraña, Angélica León, and Ariel Sánchez (ESO La Silla) for the inspiring conversations.

\section{References}

Aller, A., Lillo-Box, J., Jones, D., Miranda, L. F., \& Barceló Forteza, S. 2020, A\&A, 635, A128

Almenara, J. M., Astudillo-Defru, N., Bonfils, X., et al. 2015, A\&A, 581, L7

Almenara, J. M., Díaz, R. F., Dorn, C., Bonfils, X., \& Udry, S. 2018, MNRAS, 478,460

Ambikasaran, S., Foreman-Mackey, D., Greengard, L., Hogg, D. W., \& O’Neil, M. 2015, IEEE Trans. Pattern Anal. Mach. Intell., 38, 252

Anderson, O. L., Dubrovinsky, L., Saxena, S. K., \& LeBihan, T. 2001, Geophys. Res. Lett., 28, 399

Anglada-Escudé, G., \& Butler, R. P. 2012, ApJS, 200, 15

Barragán, O., Gandolfi, D., \& Antoniciello, G. 2019, MNRAS, 482, 1017

Batalha, N. E., Mandell, A., Pontoppidan, K., et al. 2017, PASP, 129, 064501

Berger, T. A., Huber, D., Gaidos, E., van Saders, J. L., \& Weiss, L. M. 2020, AJ, 160,108

Berta, Z. K., Irwin, J., Charbonneau, D., Burke, C. J., \& Falco, E. E. 2012, AJ, 144,145

Birch, F. 1952, J. Geophys. Res., 57, 227

Bluhm, P., Luque, R., Espinoza, N., et al. 2020, A\&A, 639, A132

Briceno, C., Hartmann, L. W., Stauffer, J. R., et al. 1997, AJ, 113, 740

Brown, T. M., Baliber, N., Bianco, F. B., et al. 2013, PASP, 125, 1031

Buchner, J., Georgakakis, A., Nandra, K., et al. 2014, A\&A, 564, A125

Carrillo, A., Hawkins, K., Bowler, B. P., Cochran, W., \& Vanderburg, A. 2020, MNRAS, 491, 4365

Casagrande, L., \& VandenBerg, D. A. 2018, MNRAS, 479, L102

Chabrier, G., Mazevet, S., \& Soubiran, F. 2019, ApJ, 872, 51

Chen, H., \& Rogers, L. A. 2016, ApJ, 831, 180

Ciardi, D. R., Beichman, C. A., Horch, E. P., \& Howell, S. B. 2015, ApJ, 805, 16

Cifuentes, C., Caballero, J. A., Cortés-Contreras, M., et al. 2020, A\&A, 642 , A115

Cloutier, R., \& Menou, K. 2020, AJ, 159, 211

Cloutier, R., Astudillo-Defru, N., Bonfils, X., et al. 2019, A\&A, 629, A111

Cloutier, R., Rodriguez, J. E., Irwin, J., et al. 2020, AJ, 160, 22 
R. Luque et al.: A multi-planetary system around TOI-776

Collins, K. A., Kielkopf, J. F., Stassun, K. G., \& Hessman, F. V. 2017, AJ, 153,

Crossfield, I. J. M., Petigura, E., Schlieder, J. E., et al. 2015, ApJ, 804, 10

Damasso, M., Bonomo, A. S., Astudillo-Defru, N., et al. 2018, A\&A, 615, A69

Demory, B. O., Pozuelos, F. J., Gómez Maqueo Chew, Y., et al. 2020, A\&A, 642, A49

Díez Alonso, E., González Hernández, J. I., Suárez Gómez, S. L., et al. 2018, MNRAS, 480, L1

Díez Alonso, E., Caballero, J. A., Montes, D., et al. 2019, A\&A, 621, A126

Drake, A. J., Graham, M. J., Djorgovski, S. G., et al. 2014, ApJS, 213, 9

Dressing, C. D., \& Charbonneau, D. 2013, ApJ, 767, 95

Dressing, C. D., \& Charbonneau, D. 2015, ApJ, 807, 45

Dumusque, X., Bonomo, A. S., Haywood, R. D., et al. 2014, ApJ, 789, 154

Elkins-Tanton, L. T., \& Seager, S. 2008, ApJ, 685, 1237

Espinoza, N. 2018, Res. Notes Amer. Astron. Soc., 2, 209

Espinoza, N., \& Jordán, A. 2015, MNRAS, 450, 1879

Espinoza, N., Kossakowski, D., \& Brahm, R. 2019, MNRAS, 490, 2262

Fei, Y., Mao, H., \& Hemley, R. J. 1993, J. Chem. Phys., 99, 5369

Feistel, R., \& Wagner, W. 2006, J. Phys. Chem. Ref. Data, 35, 1021

Feroz, F., Hobson, M. P., \& Bridges, M. 2009, MNRAS, 398, 1601

Foreman-Mackey, D., Agol, E., Ambikasaran, S., \& Angus, R. 2017, celerite: Scalable 1D Gaussian Processes in $\mathrm{C}++$, Python, and Julia

France, K., Loyd, R. O. P., Youngblood, A., et al. 2016, ApJ, 820, 89

French, M., Mattsson, T. R., Nettelmann, N., \& Redmer, R. 2009, Phys. Rev. B, 79, 054107

Fulton, B. J., \& Petigura, E. A. 2018, AJ, 156, 264

Fulton, B. J., Petigura, E. A., Howard, A. W., et al. 2017, AJ, 154, 109

Fulton, B. J., Petigura, E. A., Blunt, S., \& Sinukoff, E. 2018, PASP, 130, 044504

Gaia Collaboration (Brown, A. G. A., et al.) 2018, A\&A, 616, A1

Gaidos, E., Mann, A. W., Lépine, S., et al. 2014, MNRAS, 443, 2561

Ginzburg, S., Schlichting, H. E., \& Sari, R. 2018, MNRAS, 476, 759

Greene, T. P., Line, M. R., Montero, C., et al. 2016, ApJ, 817, 17

Güdel, M., Audard, M., Reale, F., Skinner, S. L., \& Linsky, J. L. 2004, A\&A, 416,713

Gupta, A., \& Schlichting, H. E. 2019, MNRAS, 487, 24

Gupta, A., \& Schlichting, H. E. 2020, MNRAS, 493, 792

Hadden, S., \& Lithwick, Y. 2017, AJ, 154, 5

Hamann, A., Montet, B. T., Fabrycky, D. C., Agol, E., \& Kruse, E. 2019, AJ, 158,133

Hardegree-Ullman, K. K., Zink, J. K., Christiansen, J. L., et al. 2020, ApJS, 247, 28

Hatzes, A. P. 2016, Astrophys. Space Sci. Lib., 428, 3

Hatzes, A. P., \& Rauer, H. 2015, ApJ, 810, L25

Hébrard, E., Dobrijevic, M., Loison, J.-C., Bergeat, A., \& Hickson, K. 2012, A\&A, 541, A21

Hirano, T., Dai, F., Gandolfi, D., et al. 2018, AJ, 155, 127

Howe, A. R., Burrows, A., \& Verne, W. 2014, ApJ, 787, 173

Howell, S. B., Everett, M. E., Sherry, W., Horch, E., \& Ciardi, D. R. 2011, AJ, 142,19

Irwin, J., Irwin, M., Aigrain, S., et al. 2007, MNRAS, 375, 1449

Irwin, J. M., Berta-Thompson, Z. K., Charbonneau, D., et al. 2015, in Cambridge Workshop on Cool Stars, Stellar Systems, and the Sun (San Francisco: ASP Books), 18, 767

Jenkins, J. M., Twicken, J. D., McCauliff, S., et al. 2016, Proc. SPIE, 9913, 99133E

Jensen, E. 2013, Astrophys. Source Code Libr. [record ascl : 1306.007]

Jin, S., \& Mordasini, C. 2018, ApJ, 853, 163

Jin, S., Mordasini, C., Parmentier, V., et al. 2014, ApJ, 795, 65

Johnstone, C. P., Güdel, M., Brott, I., \& Lüftinger, T. 2015, A\&A, 577, A28

Jontof-Hutter, D., Ford, E. B., Rowe, J. F., et al. 2016, ApJ, 820, 39

Journaux, B., Brown, J. M., Pakhomova, A., et al. 2020, J. Geophys. Res. Planets, 125, e06176

Karki, B. B., Wentzcovitch, R. M., de Gironcoli, S., \& Baroni, S. 2000, Phys Rev. B, 62, 14750

Kawashima, Y., \& Rugheimer, S. 2019, AJ, 157, 213

Kempton, E. M.-R., Bean, J. L., Louie, D. R., et al. 2018, PASP, 130, 114401

Kipping, D. M. 2013, MNRAS, 435, 2152

Kislyakova, K. G., Lammer, H., Holmström, M., et al. 2013, Astrobiology, 13, 1030

Kislyakova, K. G., Johnstone, C. P., Odert, P., et al. 2014, A\&A, 562, A116

Klotz, S., Komatsu, K., Kagi, H., et al. 2017, Phys. Rev. B, 95, 174111

Kochanek, C. S., Shappee, B. J., Stanek, K. Z., et al. 2017, PASP, 129, 104502

Korth, J. 2020, PhD thesis, Universität zu Köln, Germany

Kosiarek, M. R., Crossfield, I. J. M., Hardegree-Ullman, K. K., et al. 2019, AJ, 157, 97

Kreidberg, L. 2015, PASP, 127, 1161

Kreidberg, L., Bean, J. L., Désert, J.-M., et al. 2014, Nature, 505, 69

Kreidberg, L., Koll, D. D., Morley, C., et al. 2019, Nature, 573, 87
Küker, M., Rüdiger, G., Olah, K., \& Strassmeier, K. G. 2019, A\&A, 622, A40 Lam, K. W. F., Korth, J., Masuda, K., et al. 2020, AJ, 159, 120

Lammer, H., Güdel, M., Kulikov, Y., et al. 2012, Earth Planets Space, 64, 179 Lee, E. J., \& Chiang, E. 2016, ApJ, 817, 90

Lee, E. J., Chiang, E., \& Ormel, C. W. 2014, ApJ, 797, 95

Li, J., Tenenbaum, P., Twicken, J. D., et al. 2019, PASP, 131, 024506

Lillo-Box, J., Figueira, P., Leleu, A., et al. 2020, A\&A, 642, A121

Lopez, E. D., \& Fortney, J. J. 2014, ApJ, 792, 1

Lopez, E. D., \& Rice, K. 2018, MNRAS, 479, 5303

Lovis, C., \& Pepe, F. 2007, A\&A, 468, 1115

Luque, R., Pallé, E., Kossakowski, D., et al. 2019, A\&A, 628, A39

Luyten, W. J. 1974, IAU Symp., 61, 169

Madhusudhan, N., Nixon, M. C., Welbanks, L., Piette, A. A. A., \& Booth, R. A. 2020, ApJ, 891, L7

Martinez, C. F., Cunha, K., Ghezzi, L., \& Smith, V. V. 2019, ApJ, 875, 29

Mayor, M., Pepe, F., Queloz, D., et al. 2003, The Messenger, 114, 20

Molaverdikhani, K., Henning, T., \& Mollière, P. 2019a, ApJ, 883, 194

Molaverdikhani, K., Henning, T., \& Mollière, P. 2019b, ApJ, 873, 32

Molaverdikhani, K., Henning, T., \& Mollière, P. 2020, ApJ, 899, 53

Mollière, P., Wardenier, J., van Boekel, R., et al. 2019, A\&A, 627, A67

Montet, B. T., Morton, T. D., Foreman-Mackey, D., et al. 2015, ApJ, 809, 25

Mordasini, C. 2020, A\&A, 638, A52

Morton, T. D. 2015, Isochrones: Stellar model grid package (USA: NASA)

Murdoch, K. A., Hearnshaw, J. B., \& Clark, M. 1993, ApJ, 413, 349

Murray-Clay, R. A., Chiang, E. I., \& Murray, N. 2009, ApJ, 693, 23

Nelson, B. E., Ford, E. B., Buchner, J., et al. 2020, AJ, 159, 73

Niraula, P., Redfield, S., Dai, F., et al. 2017, AJ, 154, 266

Nixon, M. C., \& Madhusudhan, N. 2020, MNRAS, submitted

Nowak, G., Luque, R., Parviainen, H., et al. 2020, A\&A, 642, A173

Osborn, H. P., Santerne, A., Barros, S. C. C., et al. 2017, A\&A, 604, A19

Owen, J. E., \& Campos Estrada, B. 2020, MNRAS, 491, 5287

Owen, J. E., \& Jackson, A. P. 2012, MNRAS, 425, 2931

Owen, J. E., \& Wu, Y. 2013, ApJ, 775, 105

Pojmanski, G. 2002, Acta Astron., 52, 397

Pollacco, D. L., Skillen, I., Collier Cameron, A., et al. 2006, PASP, 118, 1407

Reddy, B. E., Lambert, D. L., \& Allende Prieto, C. 2006, MNRAS, 367, 1329

Rein, H., \& Liu, S. F. 2012, A\&A, 537, A128

Rein, H., \& Spiegel, D. S. 2015, MNRAS, 446, 1424

Riess, A. G., Casertano, S., Yuan, W., et al. 2018, ApJ, 861, 126

Rowe, J. F., Bryson, S. T., Marcy, G. W., et al. 2014, ApJ, 784, 45

Rüdiger, G., Küker, M., \& Tereshin, I. 2014, A\&A, 572, L7

Salpeter, E. E., \& Zapolsky, H. S. 1967, Phys. Rev., 158, 876

Sanz-Forcada, J., Micela, G., Ribas, I., et al. 2011, A\&A, 532, A6

Schlichting, H. E., Sari, R., \& Yalinewich, A. 2015, Icarus, 247, 8

Schweitzer, A., Passegger, V. M., Cifuentes, C., et al. 2019, A\&A, 625, A68

Scott, N. J. 2019, AAS/Division for Extreme Solar Systems Abstracts, 15, 330.15

Seager, S., Kuchner, M., Hier-Majumder, C. A., \& Militzer, B. 2007, ApJ, 669, 1279

Shuvalov, V. 2009, Meteorit. Planet. Sci., 44, 1095

Skrutskie, M. F., Cutri, R. M., Stiening, R., et al. 2006, AJ, 131, 1163

Smith, J. C., Stumpe, M. C., Van Cleve, J. E., et al. 2012, PASP, 124, 1000

Southworth, J. 2011, MNRAS, 417, 2166

Stassun, K. G., \& Torres, G. 2018, ApJ, 862, 61

Stassun, K. G., Oelkers, R. J., Pepper, J., et al. 2018, AJ, 156, 102

Steffen, J. H., Fabrycky, D. C., Ford, E. B., et al. 2012, MNRAS, 421, 2342

Stumpe, M. C., Smith, J. C., Van Cleve, J. E., et al. 2012, PASP, 124, 985

Stumpe, M. C., Smith, J. C., Catanzarite, J. H., et al. 2014, PASP, 126, 100

Teske, J. K., Ciardi, D. R., Howell, S. B., Hirsch, L. A., \& Johnson, R. A. 2018 AJ, 156, 292

Thomas, S. W., \& Madhusudhan, N. 2016, MNRAS, 458, 1330

Tokovinin, A. 2018, PASP, 130, 035002

Trotta, R. 2008, Contemp. Phys., 49, 71

Tu, L., Johnstone, C. P., Güdel, M., \& Lammer, H. 2015, A\&A, 577, L3

Twicken, J. D., Catanzarite, J. H., Clarke, B. D., et al. 2018, PASP, 130, 064502

Van Eylen, V., \& Albrecht, S. 2015, ApJ, 808, 126

Van Eylen, V., Agentoft, C., Lundkvist, M. S., et al. 2018, MNRAS, 479, 4786

Van Eylen, V., Albrecht, S., Huang, X., et al. 2019, AJ, 157, 61

Vinet, P., Rose, J. H., Ferrante, J., \& Smith, J. R. 1989, J. Phys. Conden. Matter, 1,1941

Wagner, W., \& Pruß, A. 2002, J. Phys. Chem. Ref. Data, 31, 387

Wakeford, H. R., Sing, D. K., Kataria, T., et al. 2017, Science, 356, 628

Walter, F. M., Brown, A., Mathieu, R. D., Myers, P. C., \& Vrba, F. J. 1988, AJ, 96, 297

Weiss, L. M., \& Marcy, G. W. 2014, ApJ, 783, L6

Winn, J. N. 2010, Exoplanet Transits and Occultations (Tucson: University of Arizona Press), 55

Winn, J. N., Sanchis-Ojeda, R., Rogers, L., et al. 2017, AJ, 154, 60

Woźniak, P. R., Vestrand, W. T., Akerlof, C. W., et al. 2004, AJ, 127, 2436 
Wu, Y. 2019, ApJ, 874, 91

Wyatt, M. C., Kral, Q., \& Sinclair, C. A. 2020, MNRAS, 491, 782

Xie, J.-W., Dong, S., Zhu, Z., et al. 2016, Proc. Natl. Acad. Sci., 113, 11431

Yee, S. W., Petigura, E. A., \& von Braun, K. 2017, ApJ, 836, 77

Zacharias, N., Finch, C. T., Girard, T. M., et al. 2013, AJ, 145, 44

Zechmeister, M., Kürster, M., \& Endl, M. 2009, A\&A, 505, 859

Zechmeister, M., Reiners, A., Amado, P. J., et al. 2018, A\&A, 609, A12

Zeng, L., \& Sasselov, D. 2013, PASP, 125, 227

Zeng, L., Sasselov, D. D., \& Jacobsen, S. B. 2016, ApJ, 819, 127

Zeng, L., Jacobsen, S. B., Sasselov, D. D., et al. 2019, Proc. Natl. Acad. Sci., 116, 9723

Ziegler, C., Tokovinin, A., Briceño, C., et al. 2020, AJ, 159, 19

Zinn, J. C., Pinsonneault, M. H., Huber, D., \& Stello, D. 2019, ApJ, 878, 136

${ }^{1}$ Instituto de Astrofísica de Canarias, 38205 La Laguna, Tenerife, Spain e-mail: rluque@iac.es

2 Departamento de Astrofísica, Universidad de La Laguna, 38206 La Laguna, Tenerife, Spain

3 Dipartimento di Fisica, Università degli Studi di Torino, via Pietro Giuria 1, 10125 Torino, Italy

${ }^{4}$ Max-Planck-Institut für Astronomie, Königstuhl 17, 69117 Heidelberg, Germany

${ }^{5}$ Landessternwarte, Zentrum für Astronomie der Universität Heidelberg, Königstuhl 12, 69117 Heidelberg, Germany

${ }^{6}$ Institute of Astronomy, University of Cambridge, Madingley Road, Cambridge CB3 OHA, UK

7 Department of Astronomy, University of Tokyo, 7-3-1 Hongo, Bunkyo-ky, Tokyo 113-0033, Japan

8 Thüringer Landessternwarte Tautenburg, Sternwarte 5, 07778 Tautenburg, Germany

${ }^{9}$ Rheinisches Institut für Umweltforschung an der Universität zu Köln, Aachener Strasse 209, 50931 Köln, Germany

${ }^{10}$ Center for Planetary Systems Habitability and McDonald Observatory, The University of Texas at Austin, Austin, TX 78730, USA

11 Department of Earth and Planetary Sciences, Tokyo Institute of Technology, 2-12-1 Ookayama, Meguro-ku, Tokyo 152-8551, Japan

12 Stellar Astrophysics Centre, Department of Physics and Astronomy, Aarhus University, Ny Munkegade 120, 8000 Aarhus C, Denmark

13 Sub-department of Astrophysics, Department of Physics, University of Oxford, Oxford, OX1 3RH, UK

${ }^{14}$ Cerro Tololo Inter-American Observatory/NSF's NOIRLab, Casilla 603, La Serena, Chile

15 Deutsches Zentrum für Luft- und Raumfahrt, Institut für Planetenforschung, 12489 Berlin, Rutherfordstrasse 2., Germany

16 Center for Astrophysics IHarvard \& Smithsonian, 60 Garden Street, Cambridge, MA 02138, USA
${ }^{17}$ George Mason University, 4400 University Drive, Fairfax, VA 22030 USA

18 Exoplanets and Stellar Astrophysics Laboratory, Mail Code 667, NASA Goddard Space Flight Center, 8800 Greenbelt Rd., Greenbelt MD 20771, USA

19 Department of Physics and Astronomy, University of Kansas, Lawrence, KS, USA

20 Division of Geological and Planetary Sciences, California Institute of Technology, 1200 East California Blvd, Pasadena, CA 91125, USA

${ }^{21}$ Leiden Observatory, Leiden University, 2333CA Leiden, The Netherlands

22 Department of Space, Earth and Environment, Chalmers University of Technology, Onsala Space Observatory, 43992 Onsala, Sweden

${ }^{23}$ Department of Earth, Atmospheric and Planetary Sciences, Massachusetts Institute of Technology, Cambridge, MA 02139, USA

${ }^{24}$ Department of Physics and Kavli Institute for Astrophysics and Space Research, Massachusetts Institute of Technology, Cambridge, MA 02139, USA

25 Space Science \& Astrobiology Division, NASA Ames Research Center, Moffett Field, CA 94035, USA

26 Department of Physics \& Astronomy, Swarthmore College, Swarthmore PA 19081, USA

27 Astronomical Institute, Czech Academy of Sciences, Fričova 298, 25165 Ondřejov, Czech Republic

28 Space Telescope Science Institute, Baltimore, MD, USA

29 Department of Physics and Astronomy, University of Louisville, Louisville, KY 40292, USA

30 Center for Astronomy and Astrophysics, Technical University Berlin, Hardenbergstr. 36, 10623 Berlin, Germany

31 Department of Physics and Astronomy, The University of North Carolina at Chapel Hill, Chapel Hill, NC 27599-3255, USA

32 Komaba Institute for Science, The University of Tokyo, 3-8-1 Komaba, Meguro, Tokyo 153-8902, Japan

33 JST, PRESTO, 3-8-1 Komaba, Meguro, Tokyo 153-8902, Japan

34 Astrobiology Center, 2-21-1 Osawa, Mitaka, Tokyo 181-8588, Japan

35 Astronomy Department and Van Vleck Observatory, Wesleyan University, Middletown, CT 06459, USA

${ }^{36}$ European Southern Observatory (ESO), Alonso de Córdova 3107, Vitacura, Casilla 19001, Santiago de Chile, Chile

37 Department of Aeronautics and Astronautics, Massachusetts Institute of Technology, 77 Massachusetts Avenue, Cambridge, MA 02139, USA

${ }^{38}$ Perth Exoplanet Survey Telescope, Perth, Western Australia

39 Mullard Space Science Laboratory, University College London, Holmbury St. Mary, Dorking, Surrey, RH5 6NT, UK

40 Department of Astrophysical Sciences, Princeton University, 4 Ivy Lane, Princeton, NJ 08544, USA

${ }^{41}$ Dunlap Institute for Astronomy and Astrophysics, University of Toronto, 50 St. George Street, Toronto, Ontario M5S 3H4, Canada 


\section{Appendix A: Joint fit priors}

Table A.1. Priors used for the models presented in Sect. 5 using juliet.

\begin{tabular}{|c|c|c|c|}
\hline Parameter name & Prior & Units & Description \\
\hline \multicolumn{4}{|c|}{ Stellar parameters } \\
\hline$\rho_{\star}$ & $\mathcal{N}\left(5300,1500^{2}\right)$ & $\mathrm{kg} \mathrm{m}^{-3}$ & Stellar density \\
\hline \multicolumn{4}{|c|}{ Planet parameters } \\
\hline$P_{\mathrm{b}}$ & $\mathcal{N}\left(8.24,0.05^{2}\right)$ & $\mathrm{d}$ & Period of planet $b$ \\
\hline$P_{\mathrm{c}}$ & $\mathcal{N}\left(15.65,0.05^{2}\right)$ & $\mathrm{d}$ & Period of planet $\mathrm{c}$ \\
\hline$t_{0, \mathrm{~b}}-2450000$ & $\mathcal{N}\left(8571.41,0.01^{2}\right)$ & $\mathrm{d}$ & Transit-center time of planet $b$ \\
\hline$t_{0, \mathrm{c}}-2450000$ & $\mathcal{N}\left(8572.60,0.01^{2}\right)$ & $\mathrm{d}$ & Transit-center time of planet c \\
\hline$r_{1, \mathrm{~b}}$ & $\mathcal{U}(0,1)$ & $\ldots$ & Parameterization for $p$ and $b$ of planet $\mathrm{b}$ \\
\hline$r_{2, \mathrm{~b}}$ & $\mathcal{U}(0,1)$ & $\ldots$ & Parameterization for $p$ and $b$ of planet $\mathrm{b}$ \\
\hline$r_{1, \mathrm{c}}$ & $\mathcal{U}(0,1)$ & $\ldots$ & Parameterization for $p$ and $b$ of planet $\mathrm{c}$ \\
\hline$r_{2, \mathrm{c}}$ & $\mathcal{U}(0,1)$ & $\ldots$ & Parameterization for $p$ and $b$ of planet c \\
\hline$K_{\mathrm{b}}$ & $\mathcal{U}(0,20)$ & $\mathrm{m} \mathrm{s}^{-1}$ & RV semi-amplitude of planet $b$ \\
\hline$K_{\mathrm{c}}$ & $\mathcal{U}(0,20)$ & $\mathrm{m} \mathrm{s}^{-1}$ & RV semi-amplitude of planet $\mathrm{c}$ \\
\hline$e_{\mathrm{b}}$ & $\mathcal{B}(1.52,29)$ & $\ldots$ & Eccentricity of planet $b$ \\
\hline$e_{\mathrm{c}}$ & $\mathcal{B}(1.52,29)$ & $\ldots$ & Eccentricity of planet c \\
\hline$\omega_{\mathrm{b}}$ & $\mathcal{U}(-180,180)$ & $\operatorname{deg}$ & Argument of periastron of planet $b$ \\
\hline$\omega_{\mathrm{c}}$ & $\mathcal{U}(-180,180)$ & $\operatorname{deg}$ & Argument of periastron of planet c \\
\hline \multicolumn{4}{|c|}{ Photometry parameters } \\
\hline$\sigma_{\mathrm{TESS}}$ & $\mathcal{J}(1,1000)$ & ppm & Extra jitter term for TESS \\
\hline$D_{\text {TESS }}$ & 1.0 (fixed) & $\ldots$ & Dilution factor for TESS \\
\hline$M_{\mathrm{TESS}}$ & 0.0 (fixed) & ppm & Relative flux offset for TESS \\
\hline$q_{1, \mathrm{TESS}}$ & $\mathcal{U}(0,1)$ & $\ldots$ & Quadratic limb darkening parameterization for TESS \\
\hline$q_{2, \mathrm{TESS}}$ & $\mathcal{U}(0,1)$ & $\ldots$ & Quadratic limb darkening parameterization for TESS \\
\hline$\sigma_{\text {LCO-CTIO }}$ & $\mathcal{J}\left(10,10^{5}\right)$ & ppm & Extra jitter term for LCO-CTIO \\
\hline$M_{\mathrm{LCO}-\mathrm{CTIO}}$ & $\mathcal{N}\left(0,0.01^{2}\right)$ & ppm & Relative flux offset for LCO-CTIO \\
\hline$\theta_{\text {LCO-CTIO }}$ & $\mathcal{U}(-1.0,1.0)$ & $\ldots$ & Airmass regression coefficients for LCO-CTIO \\
\hline$q_{1, \mathrm{LCO}-\mathrm{CTIO}}$ & $\mathcal{U}(0,1)$ & $\ldots$ & Linear limb darkening parameterization for LCO-CTIO \\
\hline$\sigma_{\text {LCO-SAAO }}$ & $\mathcal{J}\left(10,10^{5}\right)$ & ppm & Extra jitter term for LCO-SAAO \\
\hline$M_{\text {LCO-SAAO }}$ & $\mathcal{N}\left(0,0.01^{2}\right)$ & ppm & Relative flux offset for LCO-SAAO \\
\hline$\theta_{\text {LCO-SAAO }}$ & $\mathcal{U}(-1.0,1.0)$ & $\ldots$ & Airmass regression coefficients for LCO-SAAO \\
\hline$q_{1, \mathrm{LCO}-\mathrm{SAAO}}$ & $\mathcal{U}(0,1)$ & $\ldots$ & Linear limb darkening parameterization for LCO-SAAO \\
\hline$\sigma_{\mathrm{LCO}-\mathrm{SSO}}$ & $\mathcal{J}\left(10,10^{5}\right)$ & ppm & Extra jitter term for LCO-SSO \\
\hline$M_{\mathrm{LCO}-\mathrm{SSO}}$ & $\mathcal{N}\left(0,0.01^{2}\right)$ & ppm & Relative flux offset for LCO-SSO \\
\hline$\theta_{\mathrm{LCO}-\mathrm{SSO}}$ & $\mathcal{U}(-1.0,1.0)$ & $\ldots$ & Airmass regression coefficients for LCO-SSO \\
\hline$q_{1, \mathrm{LCO}-\mathrm{SSO}}$ & $\mathcal{U}(0,1)$ & $\ldots$ & Linear limb darkening parameterization for LCO-SSO \\
\hline$\sigma_{\text {MEarth }}$ & $\mathcal{J}\left(10,10^{5}\right)$ & ppm & Extra jitter term for MEarth \\
\hline$M_{\text {MEarth }}$ & $\mathcal{N}\left(0,0.01^{2}\right)$ & ppm & Relative flux offset for MEarth \\
\hline$q_{1, \mathrm{MEarth}}$ & $\mathcal{U}(0,1)$ & $\ldots$ & Linear limb darkening parameterization for MEarth \\
\hline \multicolumn{4}{|c|}{$R V$ parameters } \\
\hline$\mu_{\text {HARPS }}$ & $\mathcal{U}(-100,100)$ & $\mathrm{m} \mathrm{s}^{-1}$ & Systemic velocity for HARPS \\
\hline$\sigma_{\text {HARPS }}$ & $\mathcal{J}(0.1,100)$ & $\mathrm{m} \mathrm{s}^{-1}$ & Extra jitter term for HARPS \\
\hline \multicolumn{4}{|c|}{ GP hyperparameters and additional sinusoid } \\
\hline$\sigma_{\mathrm{GP}, \mathrm{TESS}}$ & $\mathcal{J}\left(10^{-2}, 10^{6}\right)$ & ppm & Amplitude of GP component for TESS \\
\hline$T_{\mathrm{GP}, \mathrm{TESS}}$ & $\mathcal{J}\left(10^{-6}, 10^{4}\right)$ & $\mathrm{d}$ & Length scale of GP component for TESS \\
\hline$K$ & $\mathcal{U}(0,20)$ & $\mathrm{m} \mathrm{s}^{-1}$ & RV semi-amplitude of the additional sinusoid \\
\hline$t_{0}-2450000$ & $\mathcal{U}(8575.0,8655.0)$ & $\mathrm{d}$ & Transit-center time of the additional sinusoid \\
\hline$P$ & $\mathcal{N}\left(35.0,10.0^{2}\right)$ & $\mathrm{d}$ & Period of the additional sinusoid \\
\hline
\end{tabular}

Notes. The prior labels of $\mathcal{N}, \mathcal{U}, \mathcal{B}$, and $\mathcal{J}$ represent normal, uniform, Beta, and Jeffrey's distributions. The parameterization for $(p, b)$ using $\left(r_{1}, r_{2}\right)$ (Espinoza 2018) and the linear $\left(q_{1}\right)$ and quadratic $\left(q_{1}, q_{2}\right)$ limb darkening parameterization (Kipping 2013) are both described in Sect. 5.2.1. 
Appendix B: HARPS RV measurements and spectral line indicators

Table B.1. Serval extraction.

\begin{tabular}{crrrrrr}
\hline \hline $\mathrm{BJD}_{\mathrm{TBD}}-2457000$ & $\mathrm{RV}\left(\mathrm{m} \mathrm{s}^{-1}\right)$ & $\sigma_{\mathrm{RV}}\left(\mathrm{m} \mathrm{s}^{-1}\right)$ & $\mathrm{CRX}\left(\mathrm{m} \mathrm{s}^{-1} \mathrm{~Np}^{-1}\right)$ & $\sigma_{\mathrm{CRX}}\left(\mathrm{m} \mathrm{s}^{-1} \mathrm{~Np}^{-1}\right)$ & $\mathrm{dLW}\left(\mathrm{m}^{2} \mathrm{~s}^{-2}\right)$ & $\sigma_{\mathrm{dLW}}\left(\mathrm{m}^{2} \mathrm{~s}^{-2}\right)$ \\
\hline 1884.75667 & 4.3 & 3.1 & -4.0 & 26.4 & -3.4 & 4.0 \\
1886.88043 & -0.2 & 1.6 & -4.7 & 13.3 & -15.1 & 2.0 \\
1887.79526 & 2.2 & 1.9 & 10.9 & 15.8 & -14.7 & 2.1 \\
1888.83087 & 1.2 & 1.3 & -12.3 & 10.7 & -13.6 & 1.9 \\
1889.79811 & 1.2 & 1.6 & 5.1 & 12.9 & -12.1 & 2.5 \\
1890.80821 & 7.3 & 2.4 & -2.6 & 19.2 & -15.0 & 3.0 \\
1894.81627 & -0.2 & 2.1 & 25.4 & 16.8 & -23.2 & 2.9 \\
1898.85272 & 4.5 & 1.4 & -3.7 & 11.2 & -25.8 & 1.5 \\
1899.86392 & 9.3 & 1.5 & 14.7 & 12.0 & -24.8 & 2.5 \\
1900.84340 & 6.0 & 1.4 & -12.9 & 11.0 & -24.4 & 1.9 \\
1902.80747 & 0.5 & 1.5 & 14.9 & 12.2 & -27.4 & 1.7 \\
1903.81551 & 0.2 & 2.0 & -4.2 & 16.2 & -15.2 & 1.8 \\
1910.81439 & 5.0 & 1.3 & -1.8 & 10.4 & -19.5 & 1.8 \\
1911.72654 & 8.3 & 1.5 & 8.6 & 12.4 & -23.4 & 2.3 \\
1912.77545 & 10.0 & 1.5 & -7.8 & 11.8 & -20.5 & 2.0 \\
1914.81139 & 10.9 & 1.5 & 13.4 & 12.2 & -23.1 & 1.8 \\
1915.74379 & 7.6 & 1.2 & 0.3 & 9.7 & -25.2 & 1.4 \\
1916.69654 & 6.0 & 1.3 & 8.3 & 10.8 & -19.5 & 1.6 \\
1918.78064 & -0.2 & 1.3 & 13.0 & 10.4 & -7.3 & 1.8 \\
1919.64336 & 3.2 & 1.4 & -0.8 & 11.0 & -16.8 & 1.8 \\
1924.80573 & 0.4 & 1.4 & 5.0 & 11.2 & -16.3 & 1.8 \\
1925.68888 & 2.7 & 1.3 & -13.1 & 10.6 & -20.7 & 1.8 \\
1925.83889 & -0.5 & 1.5 & -19.7 & 12.0 & -20.7 & 1.5 \\
1926.79127 & 0.4 & 1.3 & 3.8 & 10.7 & -18.8 & 2.2 \\
1927.83074 & 1.8 & 1.6 & -14.7 & 12.8 & -16.6 & 1.9 \\
1928.78734 & 2.9 & 1.3 & -2.8 & 10.3 & -7.4 & 2.4 \\
1929.76211 & 5.2 & 1.7 & -18.0 & 13.5 & -7.2 & 2.2 \\
1930.83118 & 6.0 & 1.6 & -10.0 & 13.2 & -7.1 & 2.4 \\
1931.76389 & 7.3 & 1.4 & -17.5 & 11.4 & &
\end{tabular}


Table B.2. TERRA extraction.

\begin{tabular}{crcccccc}
\hline \hline $\mathrm{BJD}_{\mathrm{TBD}}-2457000$ & $\mathrm{RV}\left(\mathrm{m} \mathrm{s}^{-1}\right)$ & $\sigma_{\mathrm{RV}}\left(\mathrm{m} \mathrm{s}^{-1}\right)$ & $\mathrm{H}_{\alpha}$ & $\mathrm{S}-\mathrm{index}$ & $\sigma_{\mathrm{S}-\text { index }}$ & $\mathrm{NaD}_{1}$ & $\mathrm{NaD}_{2}$ \\
\hline 1884.75667 & 0.7 & 3.2 & 0.353 & 1.501 & 0.025 & 1.050 & 0.783 \\
1886.88043 & -3.1 & 1.8 & 0.345 & 1.555 & 0.018 & 1.056 & 0.800 \\
1887.79526 & -2.4 & 1.6 & 0.358 & 1.380 & 0.012 & 1.046 & 0.792 \\
1888.83087 & -3.5 & 1.4 & 0.357 & 1.391 & 0.012 & 1.044 & 0.795 \\
1889.79811 & -2.0 & 1.4 & 0.315 & 1.496 & 0.013 & 1.041 & 0.789 \\
1890.80821 & 1.5 & 2.1 & 0.336 & 1.508 & 0.015 & 1.047 & 0.797 \\
1894.81627 & -5.6 & 1.8 & 0.346 & 1.407 & 0.016 & 1.057 & 0.787 \\
1898.85272 & 1.3 & 1.4 & 0.361 & 1.356 & 0.014 & 1.055 & 0.797 \\
1899.86392 & 5.7 & 1.5 & 0.350 & 1.399 & 0.016 & 1.060 & 0.798 \\
1900.84340 & 2.6 & 1.6 & 0.364 & 1.336 & 0.015 & 1.057 & 0.799 \\
1902.80747 & -3.2 & 1.4 & 0.357 & 1.340 & 0.013 & 1.063 & 0.796 \\
1903.81551 & -4.0 & 1.2 & 0.366 & 1.342 & 0.018 & 1.060 & 0.799 \\
1910.81439 & 3.6 & 1.2 & 0.339 & 1.445 & 0.014 & 1.053 & 0.799 \\
1911.72654 & 5.3 & 1.5 & 0.349 & 1.394 & 0.013 & 1.049 & 0.800 \\
1912.77545 & 5.6 & 1.4 & 0.315 & 1.559 & 0.015 & 1.054 & 0.790 \\
1914.81139 & 6.5 & 1.6 & 0.341 & 1.460 & 0.018 & 1.056 & 0.796 \\
1915.74379 & 4.6 & 1.0 & 0.324 & 1.462 & 0.013 & 1.056 & 0.792 \\
1916.69654 & 2.8 & 1.1 & 0.348 & 1.368 & 0.011 & 1.059 & 0.801 \\
1918.78064 & -2.5 & 1.3 & 0.359 & 1.298 & 0.013 & 1.066 & 0.804 \\
1919.64336 & -0.3 & 1.2 & 0.356 & 1.338 & 0.012 & 1.061 & 0.794 \\
1924.80573 & -3.3 & 1.3 & 0.361 & 1.246 & 0.015 & 1.054 & 0.798 \\
1925.68888 & -2.1 & 1.2 & 0.375 & 1.286 & 0.012 & 1.059 & 0.805 \\
1925.83889 & -4.3 & 1.5 & 0.369 & 1.267 & 0.014 & 1.054 & 0.789 \\
1926.79127 & -2.5 & 1.2 & 0.365 & 1.231 & 0.014 & 1.054 & 0.797 \\
1927.83074 & 0.0 & 1.8 & 0.347 & 1.275 & 0.018 & 1.046 & 0.798 \\
1928.78734 & -1.6 & 1.3 & 0.331 & 1.391 & 0.017 & 1.047 & 0.797 \\
1929.76211 & 2.2 & 1.7 & 0.349 & 1.324 & 0.018 & 1.044 & 0.802 \\
1930.83118 & 3.3 & 1.5 & 0.356 & 1.386 & 0.020 & 1.045 & 0.782 \\
1931.76389 & 4.0 & 1.4 & 0.353 & 1.415 & 0.020 & 1.039 & 0.777 \\
\hline & & & & & & &
\end{tabular}




\section{Appendix C: Corner plots}

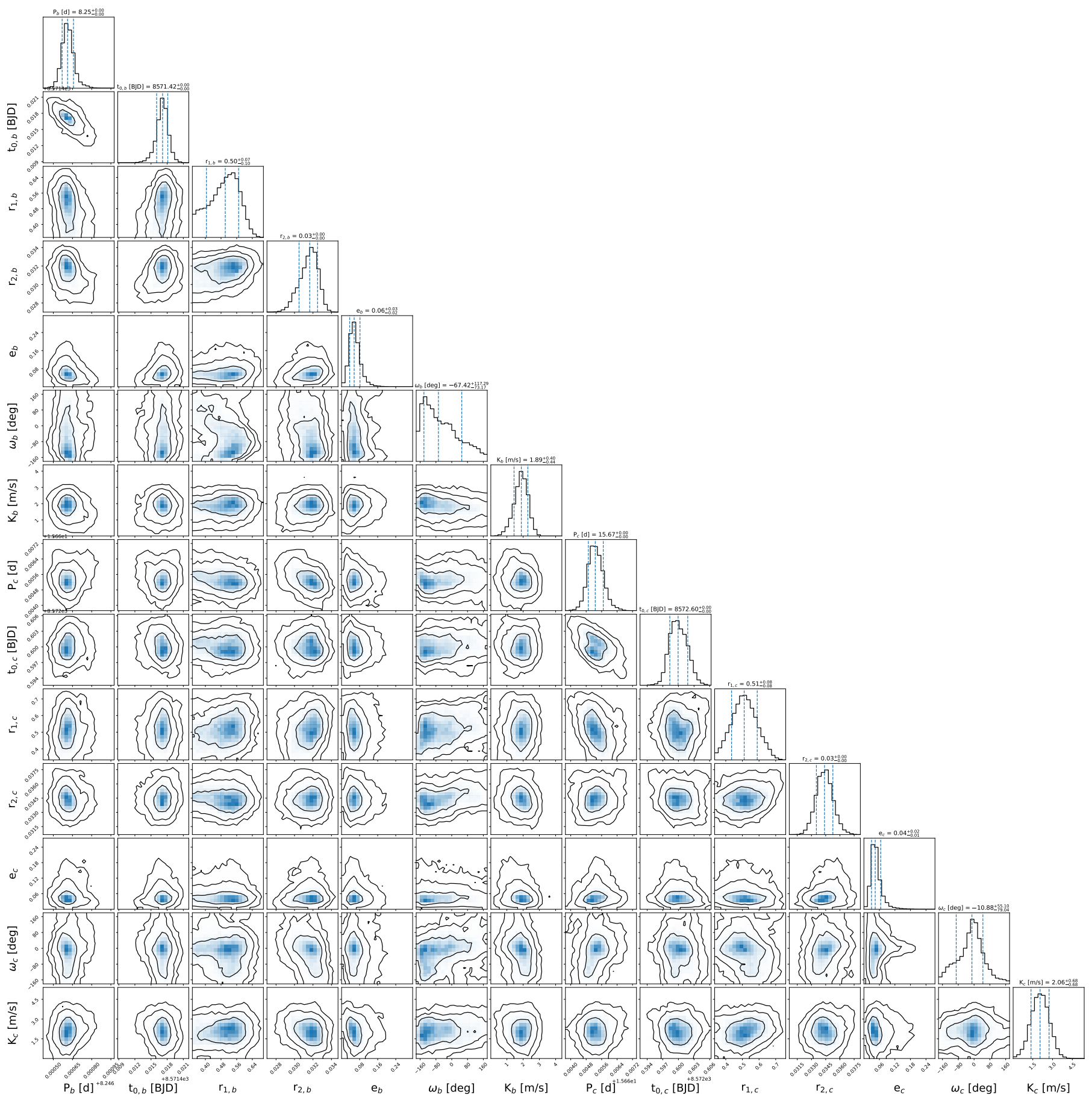

Fig. C.1. Posterior distributions of the orbital parameters of the TOI-776 system. Each panel contains $\sim 220000$ samples. The top panels of the corner plot show the probability density distributions of each orbital parameter. The vertical dashed lines indicate the 16th, 50th, and the 84th percentiles of the samples. Contours are drawn to improve the visualization of the 2D histograms and indicate the 68.3, 95.5, and 99.7\% confidence interval levels (i.e., $1 \sigma, 2 \sigma$, and $3 \sigma$ ). 